\title{
Development of Experimental Brachytherapy Dosimetry Using Monte Carlo Simulations for Detector Characterization
}

\section{Vaiva Kaveckyte}





\title{
Development of Experimental Brachytherapy Dosimetry Using Monte Carlo Simulations for Detector Characterization
}

\author{
Vaiva Kaveckyte
}

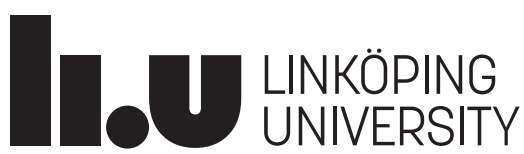

Linköping University

Department of Health, Medicine and Caring Sciences Division of Diagnostics and Specialist Medicine SE-581 83 Linköping, Sweden 
$(\mathrm{cc)}$ EY-Ne This work is licensed under a Creative Commons AttributionNonCommercial 4.0 International License.

https://creativecommons.org/licenses/by-nc/4.0/

Edition 1:1

(C) Vaiva Kaveckyte, 2021

ISBN 978-91-7929-676-6

ISSN 0345-0082

URL http://urn.kb.se/resolve?urn=urn:nbn: se:liu:diva-175192

Published articles have been reprinted with permission from the respective copyright holder.

Typeset using $\mathrm{X}_{\mathrm{H}} \mathrm{T}_{\mathrm{E}} \mathrm{X}$

Printed by LiU-Tryck, Linköping 2021 
There is nothing random. 



\section{POPULÄRVETENSKAPLIG SAMMANFATTNING}

Brachyterapi är en form av strålbehandling för att behandla cancer där man använder millimeterstora, inkapslade radioaktiva strålkällor som placeras nära eller inuti en tumör. Brachyterapi går även under namnet inre strålbehandling för att särskilja tekniken från extern strålbehandling där man bestrålar tumören utifrån med linjäracceleratorer (yttre strålbehandling). Behandlingsformen används bland annat för att behandla cancer i livmoderhals och prostata. Fördelen med inre placering av strålkällor är att den högsta absorberade dosen avges i tumören medan omkringliggande organ skonas. Vid brachyterapi för man med kirurgiska tekniker in så kallade applikatorer eller katetrar som vägleder strålkällan i patienten. Brachyterapi används för att bestråla små tumörområden eller för att höja den absorberade dosen till en del av ett större område som också ges yttre strålbehandling.

Dosimetri är läran om hur joniserande strålning deponerar energi i vävnad eller annan materia samt hur denna kvantifieras via beräkningar och mätningar med olika slags strålningsdetektorer. Framgångsrik strålbehandling kräver doser som är så höga att de riskerar att skada omkringliggande frisk vävnad allvarligt. Noggrann dosimetri är därför fundamental för alla former av strålbehandling.

Det är svårt att bestämma dosfördelningar från brachyterapikällor eftersom dosgradienter är branta och strålningens energifördelning varier med avståndet från strålkällorna. Idag görs därför få experimentella kontroller av behandlingsutrustning och mjukvara för dosberäkningar. I den här avhandlingen studeras en ny typ av strålningsdetektor som är baserad på syntetisk diamant och som utvecklats för att mäta i smala fält från linjäracceleratorer. Studierna bygger på en kombination av mätningar och den noggrannaste typen av beräkning, Monte Carlo-simulering av joniserande strålnings växelverkan med materia.

Avhandlingsarbetet visar att diamantdetektorn, tillsammans med korrektionsfaktorer som tas fram noggranna beräkningar, lämpar sig väl för mätning av absorberad dos kring ${ }^{192} \mathrm{Ir}$ strålkällor (den vanligaste isotopen för brachyterapi). Då den samtidigt är enkel att använda och endast behöver utrustning och metoder som redan finns på de flesta strålbehandlingsavdelningar öppnar resultaten för kvalitetskontroll av dosimetri för ${ }^{192} \mathrm{Ir}$, tex i form av så kallade end-to-end tester (kontroll av hela behandlingskedjan från utrustning till patientbehandling). Studierna av diamantdetektorn har kompletterats med en teoretisk studie av dosimetriska egenskaper hos materialet diamant över ett större område av energier och partikelslag (elektroner, positroner, och fotoner), till nytta för framtida studier av diamantdetektorer i flera olika former av strålbehandling.

Till slut ingår även ett arbete där Monte Carlo simuleringsmetoder för första gången har tillämpats för att karakterisera dosimetriska egenskaper hos en typ av snabba detektorer av högatomära material avsedda för in vivo mätning under pågående brachybehandling. Arbetet visar på vikten av att komplettera experiment med Monte Carlo-beräkningar för att uppnå högsta noggrannhet och för att kunna dra säkra slutsatser av experimentella fynd. 


\begin{abstract}
Brachytherapy (BT) is advantageous due to high absorbed dose conformity and possibility to deliver high dose in few fractions. It is often used for prostate and gynecological tumors as monotherapy or as a boost alongside external beam radiotherapy (EBRT). However, a number of things can compromise treatment delivery, starting from incorrect source data in a treatment planning system to malfunctioning of a treatment delivery unit. The established quality assurance (QA) covers individual aspects, such as software checks of absorbed dose calculations, mechanical checks, source dosimetry. None of them emulate treatment delivery where the planned dose could be compared with the experimentally determined values. While such practices are employed in EBRT, BT suffers from the lack of detectors that would be water-equivalent and convenient to use for regular measurements. First-choice thermoluminescence dosimeters are water-equivalent but have passive readout. Sporadic attempts to use other detectors have not led to any established practices at clinical sites. Stepping ahead, the safety of treatment delivery could be further evaluated using real-time in vivo dosimetry. If detectors were characterized with high accuracy, a reliable error detection level could be set to terminate treatments if needed. Contrary to the inphantom QA, there are detectors suitable for such applications, but their characterization is incomplete. In this thesis we address both problems.
\end{abstract}

Focusing on high-dose-rate ${ }^{192}$ Ir remote afterloading treatments, which are among the most common in BT, we investigate and propose a direct readout synthetic diamond detector for the in-phantom QA of treatment units. The detector was designed for small-field highenergy EBRT dosimetry, but our findings demonstrate its suitability for BT dosimetry. Additionally, due to the detector calibration with traceability to primary standards of absorbed dose to water of high-energy EBRT and a combination of experimental and Monte Carlo (MC) characterization, the uncertainties in the determined absorbed dose to water values were comparable to or lower than for other detectors used in BT. We complemented the investigation with a theoretical study on diamond material properties and which values (mass density, mean excitation energy, number of conduction electrons per atom) shall be used for the most faithful description of ionizing radiation interactions in diamond for MC simulations and calculations of mass electronic stopping power. The findings improve diamond dosimetry accuracy not only in BT but also in EBRT where the detectors are used.

Aiming to further contribute to the experimental BT dosimetry, we focused on high atomic number inorganic scintillators used for in vivo dosimetry: $\mathrm{ZnSe}, \mathrm{CsI}$, and $\mathrm{Al}_{2} \mathrm{O}_{3}$. These are already existing dosimeters exhibiting promising luminescence properties, but until now, their investigation has been solely experimental. MC simulations are not subject to detector positioning uncertainties which are high due to steep dose gradients and other detector response artifacts, thus we used the method to investigate the absorbed-dose energy response of detectors, its dependence on radial distance and polar angle, scatter conditions, as well as the detector design. We further clarified how error-prone high atomic number detector characterization can be if experimental and MC methods are not combined. Both have certain limitations and have to complement each other.

Though the thesis addresses two different types of detectors for two different applications, the underlying theme is to understand the detector at hand. The use of MC simulations allowed for introducing a new synthetic diamond detector into the BT dosimetry field and for improving the accuracy of in vivo dosimetry systems with inorganic scintillators. We also raised awareness to the lack of unified detector calibration and characterization practice in BT dosimetry. 


\section{List of Papers}

The thesis is based on the following papers, which are referred to in the text by their Roman numerals.

I. Kaveckyte V, Malusek A, Benmakhlouf H, Alm Carlsson G, Carlsson Tedgren $\AA 2018$ Suitability of microDiamond detectors for the determination of absorbed dose to water around high-dose-rate ${ }^{192} \mathrm{Ir}$ brachytherapy sources Med. Phys. 45 429-37

II. Kaveckyte V, Persson L, Malusek A, Benmakhlouf H, Alm Carlsson G, Carlsson Tedgren A 2020 Investigation of a synthetic diamond detector response in kilovoltage photon beams Med. Phys. 47 1268-79

III. Kaveckyte V, Carlsson Tedgren Å, Fernández-Varea J M 2021 Impact of the $I$-value of diamond on the energy deposition in different beam qualities (Conditionally accepted in Phys. Med. Biol.)

IV. Kaveckyte V, Jørgensen E B, Johansen J G and Carlsson Tedgren $\AA$ 2021 Monte Carlo characterization of high atomic number inorganic scintillators for in vivo dosimetry in ${ }^{192}$ Ir brachytherapy (Manuscript) 



\section{Acknowledgments}

Developing my inner researcher was an extraordinary experience which I will always cherish. It would have been impossible to get so far without all the support I received.

First of all, I would like to thank my principal supervisor assoc. prof. Åsa Carlsson Tedgren for taking on this Ph.D. journey with me. Your broad knowledge and ability to spot gaps in the field set a great start and continuously led to many interesting findings and contributions. You always encouraged me, let me grow and explore, and I am very glad that I got to do this with you. Not to mention that our trips together to conferences and workshops are among my very favorite not only because of all scientific discussions we had, but also personally.

Thank you to my co-supervisor dr. Alexandr Malusek. Your way of approaching questions and guidance with Monte Carlo simulations taught me a lot, and our meetings always kept me on my toes in the best way.

Thank you to my other co-supervisor dr. Hamza Benmakhlouf. Among many other insights you had, you answered countless questions about all particle spectra I showed you from Monte Carlo simulations. I was always in awe how quick your mind was and how it made me think more.

Thank you to my former co-supervisor prof. emer. Gudrun Alm Carlsson. Your vast knowledge and valuable comments made me dig deeper into the matter.

It is a great pleasure to work with like-minded people, and my collaborators are essential to this thesis.

Thank you to assoc. prof. José María Fernández-Varea. You teach by your own example, and time spent with you in Barcelona and Stockholm grew my mind immensely. I will never forget how excited I was when I first met you.

Thank you to the Aarhus scintillator group: Erik Buch Jørgensen, dr. Gustavo Kertzscher, and assoc. prof. Jacob Graversen Johansen. Your work with detectors opened my eyes to new things, and our discussions always pushed me forward. You all are a great source of inspiration for me.

Thank you to dr. Linda Persson for all detector calibrations and beyond that. You were always so professional and witty, and I feel very lucky that I got to work with you. 
I would also like thank my colleagues at the radiotherapy department at Karolinska University Hospital, and especially to Niina and Mohammed. Our talks always uplifted me, and I learned a great deal from you. You made me laugh hard, and I was always calmer knowing that you two were around the corner.

Thank you to Ricardo for bearing with me whenever I knocked on the door with my sMALL questions. Your excitement about physics and math is contagious. And let us not forget all discussions ranging from the planewave Born approximation to Richard III of England with a detour to the chi-squared test and visiting ancient Greece on the way back. The mind scatter cross section was large.

My friends sometimes had no clue what I was doing except that it involved some cross sections and detectors (diamond got special attention), but their contribution to my Ph.D. journey is immeasurable. Thank you, Ana Maria and Mattias, Caroline and Luc, Daiva and Algis, and Elisabeth, for all memories we made together. There are no words and equations to express how dear you all are to me, and I am beyond grateful that you welcomed me into your lives.

Finally and most importantly, thank you, mom and dad, for all your love and a wonderful childhood that laid the foundation for everything.

And thank you to my brother Algimantas. You are my greatest inspiration of all, and when it was hard, I always remembered you. 


\section{Contents}

Abstract iii

List of Papers $\quad$ v

Acknowledgments viii

Contents ix

1 Introduction 1

1.1 Motivation . . . . . . . . . . . . . . . . . . 1

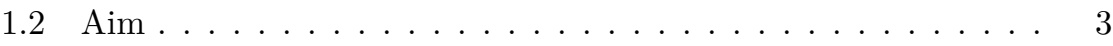

2 Background 5

2.1 Brachytherapy ...................... . . 5

2.2 Experimental brachytherapy dosimetry $\ldots \ldots \ldots \ldots \ldots$

2.3 Dosimetry basics . . . . . . . . . . . . . . . . 11

2.4 Detectors in brachytherapy . . . . . . . . . . . . . . . 14

3 Detector calibration and characterization 23

3.1 Determination of absorbed dose to water . . . . . . . . . 23

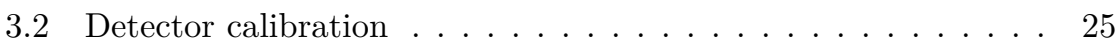

3.3 Monte Carlo simulations . . . . . . . . . . . . . . 27

4 Contributions of our research 31

4.1 Synthetic diamond detector for dosimetry in ${ }^{192}$ Ir brachytherapy 31

4.2 Svnthetic diamond response in kilovoltage photon beams . . . . 33

4.3 Diamond material properties for Monte Carlo simulations . . . 35

$4.4 \quad$ Inorganic scintillators for in vivo dosimetry in ${ }^{192}$ Ir brachytherapy 37

\begin{tabular}{|lll}
5 & Conclusions and outlook & 41
\end{tabular}

\begin{tabular}{ll}
\hline Bibliography & 43
\end{tabular} 



\section{CHAPTER}

\section{Introduction}

\subsection{Motivation}

Brachytherapy (BT) is a type of radiation therapy where a sealed radioactive source or a miniature x-ray probe is placed inside or nearby a cancerous or non-cancerous disease site. In this thesis, we focus on ${ }^{192} \mathrm{Ir}$ BT dosimetry. Due to the nature of BT treatments, namely, steep dose gradients and irradiation from within a tumor, fewer and higher-dose fractions are sufficient to achieve high tumor control probability. Treatments are prescribed either as standalone or as a boost alongside external beam radiotherapy (EBRT). At the same time, fewer fractions together with steep dose gradients pose a greater risk for under- or over-dosage of the tumor in case of a treatment delivery error, and especially in treatments using high-dose-rate (HDR) sources. Thus, high-accuracy experimental BT dosimetry is essential to not only obtain and validate source dosimetry data used for treatment planning but also for regular quality assurance (QA) of treatment units. If applicable, real-time in vivo dosimetry would further improve the assessment of absorbed dose delivered to the treatment site.

Nevertheless, measurements are challenging due to the mentioned steep dose gradients, high dose rates, non-monoenergetic spectrum of the source, and the spectrum softening as source-to-detector distance increases. A detector with an atomic number close to that of water, small volume, and negligible dose-rate dependence is the most suitable. Possessing these properties, thermoluminescence dosimeters (TLDs) have become a gold standard in experimental BT dosimetry. Their dosimetric and non-dosimetric properties have been investigated in-depth, and uncertainties related to detector response have been minimized. However, they are passive readout detectors. The signal readout process can be laborious and costly, and it requires equipment 
that is not available at many clinical sites. Therefore, while these detectors are well-suited for the determination of BT source reference data, direct readout detectors would be preferable for regular dosimetry and real-time in vivo dosimetry. Despite relatively successful attempts to use silicon-based dosimeters or nearly water-equivalent organic scintillators, these dosimeters were not adopted for regular practice either due to large absorbed-dose energy dependence or non-dosimetric properties complicating detector use.

Due to a generally low interest in the BT field until recently and the dominance of TLDs, detector development in radiotherapy dosimetry is focused on high-energy EBRT. Nevertheless, one can notice that BT measurement conditions are similar to those in fast-developing small-field high-energy photon beam dosimetry, and a synergy between the two fields can be found. Detectors adopted in small-field high-energy external beams are more complicated than those commonly used in BT (e.g., the presence of extra-cameral components and intermediate cavity size complicate the use of cavity theory in BT), but this can be remedied using Monte Carlo (MC) simulations for detector characterization in BT beams. Therefore, opening experimental BT dosimetry to new promising dosimeters, such as synthetic diamond detectors, that could fill the existing gap and tick the most important marks: direct readout, low atomic number, and negligible dose-rate dependence.

It must be noted that $\mathrm{MC}$ simulations of ionizing radiation transport are indeed widely employed for BT source characterization. However, there is a lack of awareness of the method's applicability for detector characterization. Most studies still rely on detector calibration using a clinical source and a treatment planning system (TPS). The adopted practice aims for the determination of absorbed-dose energy dependence of a detector relative to water by taking ratios of detector signal and absorbed dose to water values calculated by a TPS as a function of source-to-detector distance. However, the method does not allow for an independent check of treatment units and compromises detector characterization. Detector signal as a function of distance can change not only due to its absorbed-dose energy dependence relative to water but also due to, for instance, intrinsic energy and dose-rate dependences. Measurements are also subject to high positioning uncertainties. If MC simulations were used, the absorbed-dose energy dependence, one of the most important detector properties, could be calculated with greater accuracy. If combined with measurements, the intrinsic energy dependence and other possible detector response artifacts could be determined. It is especially important when detectors used for in vivo dosimetry are novel, and their response can be affected by multiple influence quantities.

To circumvent the detector calibration issue in BT beams, a calibration with traceability to primary standards of absorbed dose to water for highenergy photons and electrons should be used. This approach requires extensive use of MC methods to correct the absorbed-dose energy dependence of detectors between calibration and measurement beam qualities. However, it 
could bring several benefits for experimental BT dosimetry. Firstly, it would provide an independent pathway of absorbed dose to water determination in BT beams. Secondly, it would give a broader insight into detector response in different beam qualities.

\subsection{Aim}

The main aim of this thesis is to contribute to the development of experimental ${ }^{192} \mathrm{Ir}$ BT dosimetry through detector characterization with MC simulations. Firstly, by using MC as a tool to characterize and introduce a new synthetic diamond detector into the field. Secondly, by using MC to complement experimental characterization of already existing inorganic scintillators for the in vivo dosimetry.

To achieve that, we set the following research questions:

1. Is a direct readout synthetic diamond detector suitable for ${ }^{192} \mathbf{I r}$ BT dosimetry?

The detector was designed for small-field high-energy EBRT dosimetry but has properties relevant for BT dosimetry and could fill the existing gap of direct readout water-equivalent dosimeters. Thus, in Paper I we address the suitability of the detector and how calibration in terms of absorbed dose to water with traceability to primary standards of highenergy external photon beam radiotherapy allows for an independent end-to-end QA of high-dose-rate ${ }^{192}$ Ir remote afterloading units.

2. What is the intrinsic energy dependence of a synthetic diamond detector in low energy x-ray beams?

After seeing promising results in Paper $\mathbf{I}$ and having in mind a wider detector use covering low-energy BT source dosimetry and electronic BT beams, in Paper II we use MC and experimental methods to decouple the intrinsic energy dependence from the overall energy dependence. It has to be known not only to learn more about the detector, but also to improve the dosimetric accuracy when the detector is calibrated and used in different beam qualities.

3. Are we simulating radiation interactions in diamond or graphite, or neither?

Results in Paper II prompted exploration of which material properties shall be used for the most faithful and accurate MC simulation of radiation interactions in diamond. Graphite and diamond are carbon allotropes and due to their seeming similarity, diamond is often assumed to be graphite-like but with a different mass density. It is not correct, thus in Paper III we elucidate the importance of material properties 
used in MC studies and investigate how the mass density, mean excitation energy, and number of conduction electrons per atom affect energy deposition in diamond and pseudo-diamond media. The study includes a wide range of beam qualities, not only BT, due to increasing use of synthetic diamond detectors in radiotherapy dosimetry.

4. How MC studies can improve characterization of already existing high atomic number inorganic scintillators used for in vivo dosimetry in ${ }^{192}$ Ir BT?

The first three papers concern the lack of detectors for ${ }^{192} \mathrm{Ir}$ BT dosimetry, but there is also a problem of incomplete detector characterization once they are already introduced in the field. Observing a rapid development in the in vivo dosimetry followed by one-sided experimental characterization of detectors, in Paper IV we use MC simulations to characterize several existing high- $Z$ dosimeters ( $\mathrm{ZnSe}$, CsI, and $\mathrm{Al}_{2} \mathrm{O}_{3}$ ) in terms of the absorbed-dose energy dependence and what influences it under conditions relevant for in vivo dosimetry. 


\section{CHAPTER}

\section{Background}

The thesis focuses on experimental dosimetry of treatments with ${ }^{192}$ Ir sources. To avoid repetition, the term BT dosimetry refers solely to dosimetry of ${ }^{192} \mathrm{Ir}$ sources, and treatment units refer to ${ }^{192}$ Ir remote afterloading units if not specified otherwise.

\subsection{Brachytherapy}

BT is an invasive treatment where sealed radioactive sources are placed inside or nearby a treatment site (from Ancient Greek brakhús, "not far off", + therapeía, "attendance, treatment"). Depending on the treatment site and desired outcome, BT is prescribed either as monotherapy or as a boost to complement EBRT treatments. The choice of radioactive source depends on the treatment site. Cancerous eye diseases are treated using ocular plaques with photon-emitting ${ }^{125} \mathrm{I}$ sources (mean energy $28 \mathrm{keV}$ ) or ${ }^{106} \mathrm{Ru} / \mathrm{Rh} \beta$-sources (mean energy 1.4 MeV). Prostate tumors are treated either with permanent source implants or remote afterloading units. In the former case, low-dose-rate ${ }^{125} \mathrm{I}$ or ${ }^{103} \mathrm{Pd}$ seeds (photon mean energy $28 \mathrm{keV}$ and $21 \mathrm{keV}$, respectively) are placed in the prostate. In the latter case, HDR photon-emitting ${ }^{192}$ Ir sources (mean energy $350 \mathrm{keV}$ ) are the most common but pulsed-dose-rate (PDR) ${ }^{192}$ Ir sources can also be used depending on the fractionation schedule. For gynecological tumors and those in the head-and-neck area, HDR or PDR ${ }^{192} \mathrm{Ir}$ sources are used. Less often, photon-emitting ${ }^{137} \mathrm{Cs}(662 \mathrm{keV})$ and ${ }^{60} \mathrm{Co}$ (mean energy 1.25 MeV) sources are used for gynecological tumor treatment. Photon-emitting ${ }^{169} \mathrm{Yb}$ sources (mean energy $93 \mathrm{keV}$ ) have also been investigated for prostate tumor treatment (Famulari et al 2020). In electronic BT, miniature x-ray probes (generating potential from 50 to $100 \mathrm{kV}$ ) can be used 
for many treatment sites, but the most common application is breast and skin cancer treatment (Eaton 2015).

\section{Remote afterloading brachytherapy with ${ }^{192}$ Ir sources}

Remote afterloading BT with ${ }^{192} \mathrm{Ir}$ sources is used for prostate, gynecological, and head-and-neck tumor treatment. The sources are classified as high-energy photon-emitters (average energy higher than $50 \mathrm{keV}$ ) (Pérez-Calatayud et al 2012). The radionuclide has a half-life of 73.827 days and decays by $\beta^{-}$emission to excited levels of ${ }^{192} \mathrm{Pt}$ and by electron capture to excited levels of ${ }^{192}$ Os with a probability of 0.95 and 0.05 , respectively. The decay scheme is complicated, but the most important are three gamma peaks at 296, 308, $317 \mathrm{keV}$, and one at $468 \mathrm{keV}$. BT takes advantage of these gamma emissions because photon attenuation is compensated by photon scatter up to $5 \mathrm{~cm}$ distance from the source under full scatter conditions in water $(20 \mathrm{~cm}$ from the source to the edge of the scatter volume) (Taylor and Rogers 2008). As a result, a conformal absorbed dose distribution can be created within the treatment volume with a rapid dose fall-off at its periphery.

Another advantage of ${ }^{192}$ Ir sources is their high specific activity that allows for the construction of small cylindrical sources fitting into narrow treatment needles. Depending on the source design, the active core diameter ranges from 0.3 to $0.7 \mathrm{~mm}$ and the length from 1.3 to $10.0 \mathrm{~mm}$. The active core is encapsulated in a submillimeter thickness stainless steel or platinum layer and attached to a cable that is connected to a remote afterloader. Before the treatment, either metallic or plastic needles (up to 20 for interstitial treatments) are inserted into the treatment site and attached to the tubes leading to the remote afterloader. During the treatment, the source is retracted from the afterloader to a given needle where it is stepped according to the source dwell position and time pattern calculated by a TPS to achieve the desired absorbed dose distribution. For gynecological tumors, the same principle applies but instead of interstitial needles, intracavitary applicators that may have built-in needles are used. Both HDR and PDR ${ }^{192}$ Ir sources are used for the described treatments. The former has activity of approximately $400 \mathrm{GBq}$ and a dose rate higher than 2 Gy per minute at $1 \mathrm{~cm}$ from the source. The latter has about ten times lower activity and a dose rate of about 2 Gy per hour.

\subsection{Experimental brachytherapy dosimetry}

Experimental BT dosimetry can be divided into two main branches:

1. Source strength dosimetry in terms of air-kerma rate using well-type air ionization chambers. 
2. Dosimetry for source reference dataset determination and validation, inphantom QA of treatment units and in vivo measurements in terms of absorbed dose to water using probe-type detectors.

This thesis addresses detector choice and characterization for the second branch of applications where experimentally determined absorbed dose to water values are compared with TPS calculations. But first, to be able to evaluate the results and what dosimetric checks are needed, the section describes main aspects of source calibration and dosimetry, and absorbed dose calculation formalism. Afterwards, experimental BT dosimetry areas are introduced.

\section{Absorbed dose calculation formalism for brachytherapy treatment planning}

Task Group No. 43 Report (TG-43) issued by the American Association of Physicists in Medicine (AAPM) describes the absorbed dose calculation formalism implemented in TPSs and provides reference datasets for low-energy BT sources (Rivard et al 2004). Calculations for BT treatments with ${ }^{192} \mathrm{Ir}$ and other high-energy sources follow the same recommendations, but the protocol was extended and adapted to account for differences in source design and how the reference datasets shall be obtained ( $\mathrm{Li}$ et al 2007, Pérez-Calatayud et al 2012). A note must be made that there are TPSs that use model-based calculations (Beaulieu et al 2012), but they are seldom implemented in clinical use. A TPS based on the TG-43 formalism provides absorbed dose to water calculations in liquid water which is the reference medium. For high-energy sources, calculations are made in unbounded water phantoms $\left(40 \times 40 \times 40 \mathrm{~cm}^{3}\right)$ without taking into account patient anatomy, size, and location of the treatment site.

Following the formalism, the absorbed dose rate in water in a water phantom at the point of interest is calculated as follows

$$
\dot{D}(r, \theta)=S_{K} \cdot \Lambda \cdot \frac{G_{L}(r, \theta)}{G_{L}\left(r_{0}, \theta_{0}\right)} \cdot g_{L}(r) \cdot F(r, \theta),
$$

where $r$ is the distance from the center of the source to the point of interest, and $\theta$ is the polar angle between the source and the point of interest. Notations $r_{0}$ and $\theta_{0}$ refer to the reference point which is at $1 \mathrm{~cm}$ on the transverse plane, i.e., $\left(r_{0}=1 \mathrm{~cm}, \theta_{0}=90^{\circ}\right)$.

Other quantities in equation (2.1) are defined as follows:

1. The source air-kerma strength $S_{K}$ is the air-kerma rate of a source determined in vacuo at a reference distance.

2. The dose-rate constant of the source $\Lambda$ relates the source air-kerma strength $S_{K}$ to the absorbed dose rate in water at a reference point $\left(r_{0}\right.$, $\left.\theta_{0}\right)$. 
3. The geometry function $G_{L}(r, \theta)$ describes the dose fall-off due to the inverse-square law and takes into account the radioactivity distribution in a source (a point-source versus elongated source). It is used to improve tabulated dataset interpolation accuracy in a TPS.

4. The radial dose function $g_{L}(r)$ describes the dose fall-off on the transverse plane due to photon scatter and attenuation in the reference medium of unbounded liquid water phantom.

5. The anisotropy function $F(r, \theta)$ takes into account photon scatter and attenuation in the source core and its encapsulation as a function of distance and polar angle between the source center and the point of interest.

\section{Source calibration and dosimetry}

BT dosimetry chain begins with the source calibration traceable to primary standards. Although the end quantity of interest is absorbed dose to water, the starting point is the air-kerma strength $S_{K}$ or the reference air-kerma rate RAKR of the source, because there are no established primary standards for ${ }^{192}$ Ir sources in terms of absorbed dose to water. There have been promising developments for ${ }^{192} \mathrm{Ir}$ sources using water calorimetry and Fricke dosimetry (Sarfehnia and Seuntjens 2010, deAlmeida et al 2014). Uncertainties in $D_{\text {wat }}$ measured with water calorimeter were comparable to those of $S_{K}$. The difference from absorbed dose to water calculated following the air-kerma based methods (i.e., the TG-43 formalism) was $0.6 \%$, but despite that, the primary standards are still under development.

In North America, the recommended quantity for the specification of photon-emitting BT sources is $S_{K}$ (in units of U) defined by the TG- 43 formalism. In Europe, the recommended quantity is the RAKR (in units of Gy s $^{-1}$ ) defined in ICRU Reports 37 and 58 (ICRU 1985, 1997). Expressions used to obtain the two quantities are different but numerical values are the same (with the relation of units $1 \mathrm{U}=1 \mu \mathrm{Gy} \mathrm{m}^{2} \mathrm{~h}^{-1}$ ).

Primary standards of air-kerma and methods used for ${ }^{192} \mathrm{Ir}$ source calibration also depend on the region. In North America, the National Institute of Standards and Technology (NIST) uses a wide-angle free-air ionization chamber for measuring air-kerma for $250 \mathrm{kV}$ x-rays and a spherical air ionization chamber for ${ }^{137} \mathrm{Cs}$ because no primary standard exists for ${ }^{192} \mathrm{Ir}$ sources. An interpolation method is used for the determination of air-kerma rate for ${ }^{192} \mathrm{Ir}$ sources since the air-kerma weighted average energy is $397 \mathrm{keV}$, which falls in the middle between the average energy of $250 \mathrm{kV}$ and ${ }^{137} \mathrm{Cs}$ spectra. The method was originally developed by Goetsch et al (1991). Later it was shown to have defects which do not translate into significant errors (Mainegra-Hing and Rogers 2006), thus the practice remained with some improvements (Rasmussen et al 2011). The total uncertainty in $S_{K}$ following the best practice 
is $2.2 \%(k=2)$ (DeWerd et al 2011). In Europe, some calibration laboratories have traceability route to the National Physical Laboratory where a direct realization of air-kerma standards for ${ }^{192} \mathrm{Ir}$ exists. The method uses a spherical graphite-walled air ionization chamber, and the total uncertainty in the RAKR is $0.7 \%(k=2)$ (Sanders and Nutbrown 2006). The two primary standard realization methods agree within $0.3 \%$ (Soares et al 2009).

The ${ }^{192} \mathrm{Ir}$ source calibrated against the primary standard is used to calibrate well-type air-ionization chambers to establish traceability route. The chambers are used at national or secondary standard laboratories to crosscalibrate well-type chambers for the end users at clinical sites. Sources delivered to hospitals have certificates provided by the vendor where the $S_{K}$ values traceable to NIST are specified with the uncertainty of $5 \%(k=3)$. Despite the provided values, it is the duty of a medical physicist to control that they are correct. Not doing so or using an instrument that does not have calibration traceable to primary standards poses a risk for erroneous absorbed dose calculations.

Once $S_{K}$ or RAKR is measured, it must be related to the absorbed dose to water in water via the dose-rate constant $\Lambda$. For low-energy sources, $\Lambda$ must be determined using both experimental and MC methods. The average value is used in the reference datasets. For high-energy sources, MC methods alone are deemed to be sufficient. The measured $\Lambda$ values are used only to validate $\mathrm{MC}$ results and the two should agree within 1\% (Pérez-Calatayud et al 2012) .

\section{Source reference dataset dosimetry}

All quantities in equation (2.1) constitute reference datasets that are entered into a TPS for absorbed dose calculations. The data are source-specific due to differences in design (dimensions of the active core, encapsulation material and thickness) and must be determined either experimentally, or with MC methods, or both. Either type of characterization is sufficient for conventional encapsulated sources $\left({ }^{137} \mathrm{Cs}\right.$ and $\left.{ }^{192} \mathrm{Ir}\right)$ with the total uncertainty in the absorbed dose-rate distributions within $6 \%(k=2)$. For non-conventional or newly introduced sources, both MC and experimental studies should be performed ( $\mathrm{Li}$ et al 2007). To contrast, experiments and calculations are required for all low-energy photon-emitting sources (Rivard et al 2004).

When measurements are not required, MC methods are preferred since they are not susceptible to experimental uncertainties associated with detector positioning and characterization. In an MC study, Granero et al (2011) estimated that the total uncertainty in the dose-rate determined for one of the most common HDR ${ }^{192} \operatorname{Ir}$ sources is $3 \%(k=2)$ excluding the uncertainty in $S_{K}$. While uncertainties related to the approximations used for radiation transport and the underlying interaction cross section data are difficult to estimate for every given case, there are MC codes of ionizing radiation transport, 
such as PENELOPE (Salvat 2019) and EGSnrc (Kawrakow et al 2013), that are well-benchmarked even for low-energy electron transport (Sempau et al 2003, Ali and Rogers 2008). Thus, in principal, MC provides high-accuracy reference datasets for high-energy photon emitting sources. However, the reference data is calculated only for a generic source-type design, whereas the individual source dimensions and material composition may vary. Additionally, MC simulation input data is prone to user errors (two independent investigations are preferred but not required), and only measurements could validate the data. It is expected that the total expanded uncertainty in the experimentally determined dose-rate should not exceed $17 \%(k=2)$ (Li et al 2007). The value was adopted from the TG-43 formalism, which concerns low-energy photon emitting sources, and it is likely overestimated for dosimetry of high-energy photon-emitting sources.

\section{In-phantom quality assurance of ${ }^{192}$ Ir remote afterloading units}

A regular QA program of remote afterloading BT treatment units is needed to ensure safe treatment delivery. Task group No. 59 Report issued by the AAPM recommends which QA checks and how often they should be performed (Kubo et al 1998). Some countries have their national guidelines (Frenière 2018, Steenhujsen et al 2018) and there is also a practical guide issued by the European Society for Therapeutic Radiology and Oncology (Venselaar and Pérez-Calatayud 2004). The programs are similar and cover mechanical checks, software checks, radiation safety checks, but none of them encompass the entire treatment delivery chain to determine absorbed dose to water experimentally and independently from TPS calculations. Such a method would offer a quick and convenient method to ensure system integrity and could be used for inter-institutional comparison as external dosimetry audits are rare.

To contrast, regular measurements for patient-specific intensity modulated treatment plan verification are done in EBRT using either electronic portal imaging devices or special-purpose phantoms with diodes or air ionization chambers. Thus, it appears that BT does not have such practices not because the existing QA checks are deemed sufficient but rather due to the lack of suitable detectors. While patient-specific plan verification might be not achievable in BT yet, periodic end-to-end in-phantom QA of treatment units would give greater confidence in treatment delivery and ensure its integrity. Such measurements could also be used to verify model-based TPSs where data configuration is more complicated than for the ones based on the TG-43 formalism. 


\section{In vivo dosimetry of treatments with ${ }^{192}$ Ir remote afterloading units}

In vivo dosimetry for ${ }^{192} \mathrm{Ir}$ BT treatments is motivated by the same reasons as the in-phantom QA, but the former provides an advantage of error detection during the treatment (Tanderup et al 2013, Fonseca et al 2020). There have been developments in imaging systems for source position and dwell time tracking (Fonseca et al 2017, Smith et al 2018), but having a dosimetry system that allows for the determination of absorbed dose delivered to the treatment site would be preferrable. An ideal detector should be small but with high sensitivity and fast enough for time-resolved dosimetry. Various types of detectors, such as organic scintillators (Lambert et al 2006, Therriault-Proulx et al 2011, Linares Rosales et al 2020a), 2D diode arrays (Poder et al 2019), and inorganic scintillators (Andersen et al 2009, Kertzscher and Beddar 2019, Debnath et al 2021, Jørgensen et al 2021b, Jørgensen et al 2021a) have undergone the investigations and showed rather promising results. Thus, contrary to the in-phantom QA dosimetry, there is no apparent lack of suitable detectors.

On the other hand, the existing ones cannot offer high-accuracy dosimetry due to their incomplete characterization which is hitherto mainly experimental in the mentioned studies. There, assumptions are made about detector signal being proportional to the absorbed dose in the detector medium. Additionally, the detectors are calibrated using clinical sources, and a ratio of detector signal per absorbed dose to water as a function of source-to-detector distance calculated using a TPS is taken as the absorbed-dose energy dependence. But strictly speaking, such a function is not the absorbed-dose energy dependence since it may include other detector response artifacts. Though experimental measurements are essential, there is a clear need to improve in vivo dosimetry by complementing detector characterization with MC simulations.

\subsection{Dosimetry basics}

The section describes dosimetry basics that later will help to understand the choice of detector medium for BT dosimetry and methods for detector characterization. Dosimetry concerns determination of the absorbed dose in the medium of interest due to ionizing radiation interactions. In BT, treatment planning systems are based on absorbed dose to water calculations, thus the medium of interest is water. Determination of absorbed dose to water is done indirectly, by placing detector in the irradiation field at a point of interest. Ionizing radiation interacts in detector medium and deposits energy which generates detector signal. The latter must be related to the average absorbed dose in the detector medium $\bar{D}_{\text {det }}$ which is finally converted to the absorbed dose in water $D_{\text {wat }}$ at the point of measurement in the absence of the detec- 
tor. Theory describing relationship between the absorbed dose in the detector medium and the reference medium is called cavity theory.

\section{Absorbed dose}

To begin with, the absorbed dose in the medium $D_{\text {med }}$ (either detector cavity or water) can be calculated using particle (either photon or electron) fluence $\phi_{\text {med }}$ traversing the medium. If charged particle equilibrium (CPE) is established between the volume of interest and the surrounding volume (i.e, all charged particles exiting the volume of interest are replaced with identical particles entering from outside), the absorbed dose is equal to the collision kerma. Then, for a spectrum of photon energies, the relationship between the absorbed dose in the medium and the photon fluence is as follows

$$
D_{\text {med }}=K_{\text {col,med }}=\int_{0}^{E_{\text {max }}} E \cdot \phi_{\mathrm{E}}^{\text {med }} \cdot\left[\mu_{\mathrm{en}}(E) / \rho\right]_{\text {med }} \mathrm{d} E,
$$

where $\phi_{\mathrm{E}}^{\text {med }}$ is the photon fluence differential in energy in the medium, $\left[\mu_{\mathrm{en}}(E) / \rho\right]_{\text {med }}$ is the mass energy-absorption coefficient of the medium for photons with energy $E$. Here, it is also assumed that the energy deposition due to the bremsstrahlung emission by the secondary electrons is negligible in the volume of interest. When the medium is irradiated with an external beam, the CPE conditions are not fulfilled because photon attenuation is nonnegligible over the maximum secondary electron range. Instead, partial CPE is established beyond the maximum range of secondary particles where the absorbed dose in the medium is proportional, but not equal, to $K_{\text {col,med }}$ at the point of interest.

For a spectrum of charged particles, the absorbed dose in the medium is equal to the cema if $\delta$-ray equilibrium is established between the volume of interest and the surrounding volume. Then, the relationship between the absorbed dose in the medium and the charged particle fluence is as follows

$$
D_{\text {med }}=C_{\text {med }}=\int_{0}^{E_{\text {max }}} \phi_{\mathrm{E}}^{\text {med }} \cdot\left[S_{\mathrm{el}}(E) / \rho\right]_{\text {med }} \mathrm{d} E,
$$

where $\phi_{\mathrm{E}}^{\text {med }}$ is the charged particle fluence differential in energy in the medium, $\left[S_{\mathrm{el}}(E) / \rho\right]_{\text {med }}$ is the mass electronic stopping power of the medium for charged particles with energy $E$.

\section{Cavity theory}

Knowing the absorbed dose expressions, the relationship between the absorbed dose in the detector medium and the reference medium (henceforth water) can be described using cavity theory (Andreo et al 2017). The choice of cavity theory for a given case depends on the incident beam (particle type 
and energy) and cavity dimensions compared to the range of electrons depositing energy in the cavity. Two extreme cases are briefly introduced below. It will not be discussed here, but there is also the intermediate cavity theory (also known as the general, or Burlin, cavity theory) which combines equations (2.4) and (2.5) via a weighting factor.

\section{Large cavity}

When the CPE exists and the range of secondary particles produced by photons interacting in the detector medium is smaller than the detector cavity dimensions, the ratio of the absorbed dose in water and the detector medium can be determined from

$$
\frac{D_{\mathrm{wat}}}{\bar{D}_{\mathrm{det}}}=\frac{\int_{0}^{E_{\max }} E \cdot \phi_{\mathrm{E}}^{\mathrm{wat}} \cdot\left[\mu_{\mathrm{en}}(E) / \rho\right]_{\mathrm{wat}} \mathrm{d} E}{\int_{0}^{E_{\max }} E \cdot \phi_{\mathrm{E}}^{\mathrm{wat}} \cdot\left[\mu_{\mathrm{en}}(E) / \rho\right]_{\mathrm{det}} \mathrm{d} E}=\left(\frac{\bar{\mu}_{\mathrm{en}}}{\rho}\right)_{\mathrm{det}}^{\text {wat }} .
$$

Please note that the photon fluence differential in energy $\phi_{\mathrm{E}}$ refers to the water medium in both cases. A condition of negligible photon fluence perturbation due to the presence of detector cavity must be satisfied. In such a case, the quantity $D_{\text {wat }} / \bar{D}_{\text {det }}$ can be approximated as the ratio of the photon energyfluence-averaged mass energy-absorption coefficients for water and detector media.

\section{Small cavity}

When the $\delta$ ray equilibrium exists and the range of electrons traversing detector cavity is greater than its dimensions, the ratio of the absorbed dose in water and the detector medium can be determined from

$$
\frac{D_{\mathrm{wat}}}{\bar{D}_{\mathrm{det}}}=\frac{\int_{0}^{E_{\max }} \phi_{\mathrm{E}}^{\mathrm{wat}} \cdot\left[S_{\mathrm{el}}(E) / \rho\right]_{\mathrm{wat}} \mathrm{d} E}{\int_{0}^{E_{\max }} \phi_{\mathrm{E}}^{\mathrm{wat}} \cdot\left[S_{\mathrm{el}}(E) / \rho\right]_{\mathrm{det}} \mathrm{d} E}=\left(\frac{\bar{S}_{\mathrm{el}}}{\rho}\right)_{\mathrm{det}}^{\text {wat }} .
$$

Here, it is assumed that the fraction of photon-generated secondary electrons inside the cavity is negligible and that no $\delta$ rays are produced inside the cavity. Similarly to the large cavity theory, the particle fluence perturbation due to the presence of detector cavity shall be negligible. Then, the quantity $D_{\text {wat }} / \bar{D}_{\text {det }}$ can be approximated using the particle fluence-averaged mass electronic stopping powers for water and detector media. This type of cavity is referred to as the Bragg-Gray cavity, and it concerns only primary electrons. In real situations, there are energetic $\delta$ rays that escape the cavity and are not replaced by those coming from outside when the detector medium does not match the surrounding medium. That is, the $\delta$ ray equilibrium is violated. To account for such energy depositions, the Bragg-Gray cavity theory is extended to the Spencer-Attix cavity theory where a cutoff energy for charged particles is introduced. The latter theory yields a better agreement with experiments and is used for calculation of stopping-power ratios in the EBRT dosimetry. 
However, for the sake of the discussion in the thesis, it is enough to keep in mind the ideal case, i.e., the Bragg-Gray cavity theory.

\subsection{Detectors in brachytherapy}

\section{Detector properties}

Here we define main detector properties and what role they play in ${ }^{192} \mathrm{Ir}$ BT dosimetry. Ideally, detector properties would be independent of each other, and it will be assumed so unless stated otherwise. In practice, one must keep in mind that there exist interplay effects which may affect the accuracy of detector characterization.

\section{Absorbed-dose energy dependence}

The absorbed-dose energy dependence describes how the average absorbed dose in the detector medium $\bar{D}_{\text {det }}$ relates to the absorbed dose in water $D_{\text {wat }}$ in a beam quality $Q$ as follows

$$
f_{Q}=\left[\frac{D_{\text {wat }}}{\bar{D}_{\text {det }}}\right]_{Q} .
$$

The ratio can be recognized from Section 2.3 where we dealt with dosimetry basics and cavity theory. Thus, evidently absorbed-dose energy dependence is a fundamental detector property upon which the choice of the detector medium is made. From equations (2.4) and (2.5), it follows that the absorbed-dose energy dependence can be evaluated using either $\left[\mu_{\mathrm{en}}(E) / \rho\right]_{\mathrm{det}}^{\text {wat }}$ or $\left[S_{\mathrm{el}}(E) / \rho\right]_{\mathrm{det}}^{\mathrm{wat}}$, or both (intermediate cavity). The values of $\left[\mu_{\mathrm{en}}(E) / \rho\right]_{\mathrm{det}}^{\text {wat }}$ and $\left[S_{\mathrm{el}}(E) / \rho\right]_{\mathrm{det}}^{\text {wat }}$ are plotted in Figures 2.1 and 2.2, respectively, for the selected detector media (typically used in BT and investigated in the thesis).

For ${ }^{192} \operatorname{Ir}$ BT dosimetry, $\left[\mu_{\mathrm{en}}(E) / \rho\right]_{\text {det }}^{\text {wat }}$ values are most relevant because large cavity theory conditions are usually satisfied. Variation in $\left[S_{\mathrm{el}}(E) / \rho\right]_{\mathrm{det}}^{\text {wat }}$ as a function of electron kinetic energy is relatively low for all materials, thus even for dosimeters falling into the intermediate cavity theory, it is still $\left[\mu_{\mathrm{en}}(E) / \rho\right]_{\mathrm{det}}^{\mathrm{wat}}$ that determines the absorbed-dose energy dependence. For comparison, in conventional high-energy photon and electron EBRT dosimetry, the absorbed-dose energy dependence of detectors is described by $\left[S_{\mathrm{el}}(E) / \rho\right]_{\text {det }}^{\text {wat }}$.

The water-equivalence of detector medium is determined by how close its properties, namely, atomic number $Z$ and mass density $\rho$ resemble water (see Table 2.1). If they are close, particle interaction cross sections for the detector medium will be comparable to those for water. As a result, $D_{\text {wat }} / \bar{D}_{\text {det }}$ in equation (2.6) will be close to unity, and it will hold over a wide photon energy range relevant for ${ }^{192} \mathrm{Ir}$ BT dosimetry, e.g., alanine, lithium formate, diamond, polystyrene (organic scintillators), lithium flouride (typical TLD material). It 


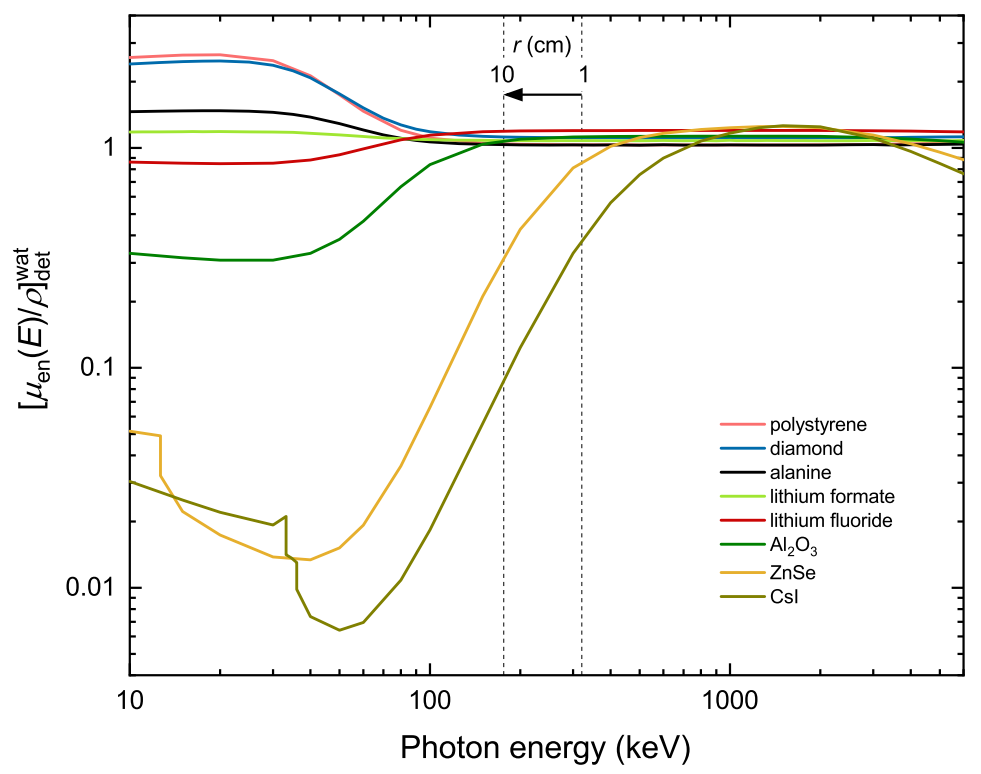

Figure 2.1: Water-to-medium ratios of mass energy-absorption coefficients $\left[\mu_{\mathrm{en}}(E) / \rho\right]_{\mathrm{det}}^{\text {wat }}$ for selected detector media as a function of photon energy. The curves were obtained from the mutren program included in PENELOPE. Vertical dashed lines correspond to the photon fluence-weighted energy range in water in the absence of the detector medium at radial distances $r$ from ${ }^{192} \mathrm{Ir}$ source in a $40 \times 40 \times 40 \mathrm{~cm}^{3}$ water phantom.

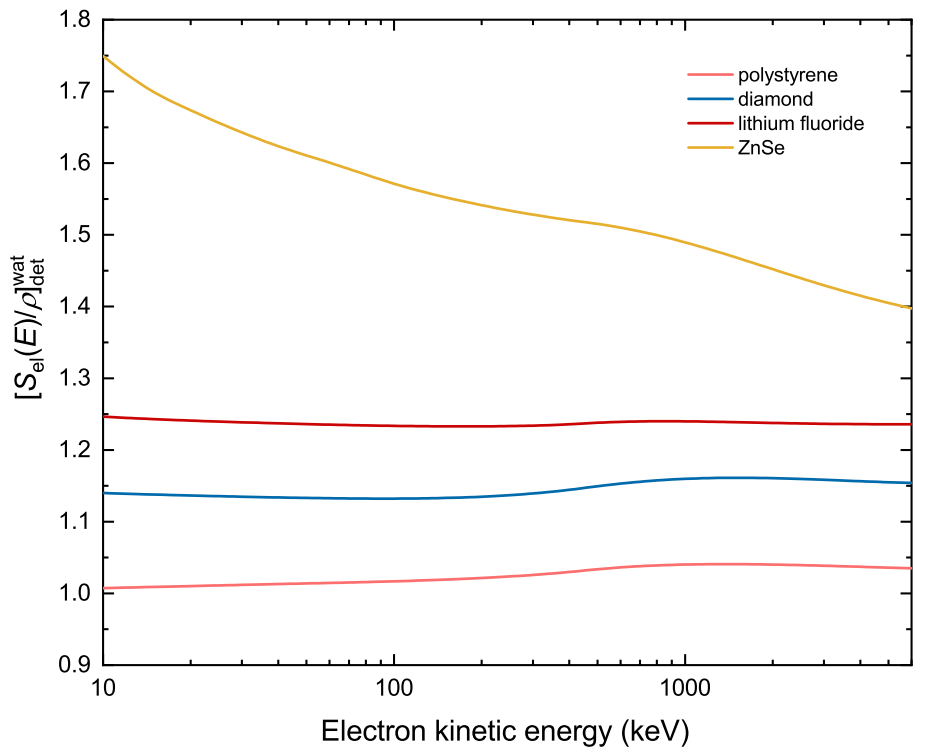

Figure 2.2: Water-to-medium ratios of mass electronic stopping powers $\left[S_{\mathrm{el}}(E) / \rho\right]_{\mathrm{det}}^{\text {wat }}$ for selected detector media as a function of electron kinetic energy. The curves were obtained from the material.exe pre-processor of PENELOPE. Not all materials from Figure 2.1 are displayed here due to similar values of $\left[S_{\mathrm{el}}(E) / \rho\right]_{\mathrm{det}}^{\text {wat }}$. 
is especially important when photon spectrum softens substantially with increasing source-to-detector distance due to the dominance of Compton scatter for the major gamma emissions (Figure 2.3).

For practical reasons, such as direct readout and higher sensitivity, high$Z$ detectors might be a preferred option (e.g., silicon, inorganic scintillators). However, due to larger cross section for the photoelectric effect $\left({ }_{\mathrm{a}} \sigma_{\mathrm{ph}}\right.$ $\propto Z^{4}$ ), such detectors over-respond compared to water. Additionally, the over-response increases with increasing distance where the fraction of low energy photons is non-negligible, and the photoelectric effect dominates $\left(\sigma_{\mathrm{ph}} \propto\right.$ $\left.E^{-3}\right)$. For such detectors, the absorbed-dose energy dependence will be not only greater for a given beam quality, but it will also vary more as a function of photon energy compared to water-equivalent detectors.

\section{Intrinsic energy dependence}

Intrinsic energy dependence of the detector relates its signal $M$ per average absorbed dose in the detector medium $\bar{D}_{\text {det }}$ in a beam quality $Q$ as follows

$$
R_{Q}=\left[\frac{M}{\bar{D}_{\mathrm{det}}}\right]_{Q} .
$$

The quantity is called the intrinsic energy dependence because it is usually used to describe the detector readout dependence on the ionization track density of secondary electrons in TLDs and other passive readout detectors. Due to the complexity of solid-state physics, these processes and their dependence on beam quality are not always well-understood. Additionally, the interplay effects of the detector response dependence on, for instance, dose-rate and accumulated dose are complicated to decouple. Thus, in practice, $R_{Q}$ can encompass the entire detector readout process from signal generation to its collection.

\section{Dose and dose-rate dependence}

A typical dose-rate in water is $2 \mathrm{~Gy} \mathrm{~min}^{-1}$ at $1 \mathrm{~cm}$ distance from an $\mathrm{HDR}{ }^{192} \mathrm{Ir}$ source, and it rapidly drops with the inverse-square of source-to-detector distance. Thus, detectors should have negligible dose-rate dependence and linear response to absorbed dose over a wide range. The dose-rate dependence is usually caused by recombination of charges created in the active volume (e.g., ion pairs in air ionization chambers or electron-hole pairs in semiconductors). For air ionization chambers, recombination correction factors are determined using methods described by well-established theories applicable in EBRT dosimetry (Andreo et al 2000 and references therein). For solid-state detectors used in BT dosimetry, the dependence is usually determined experimentally by varying the source-to-detector distance and quantifying the change in detector signal. It poses a problem since the absorbed-dose energy 

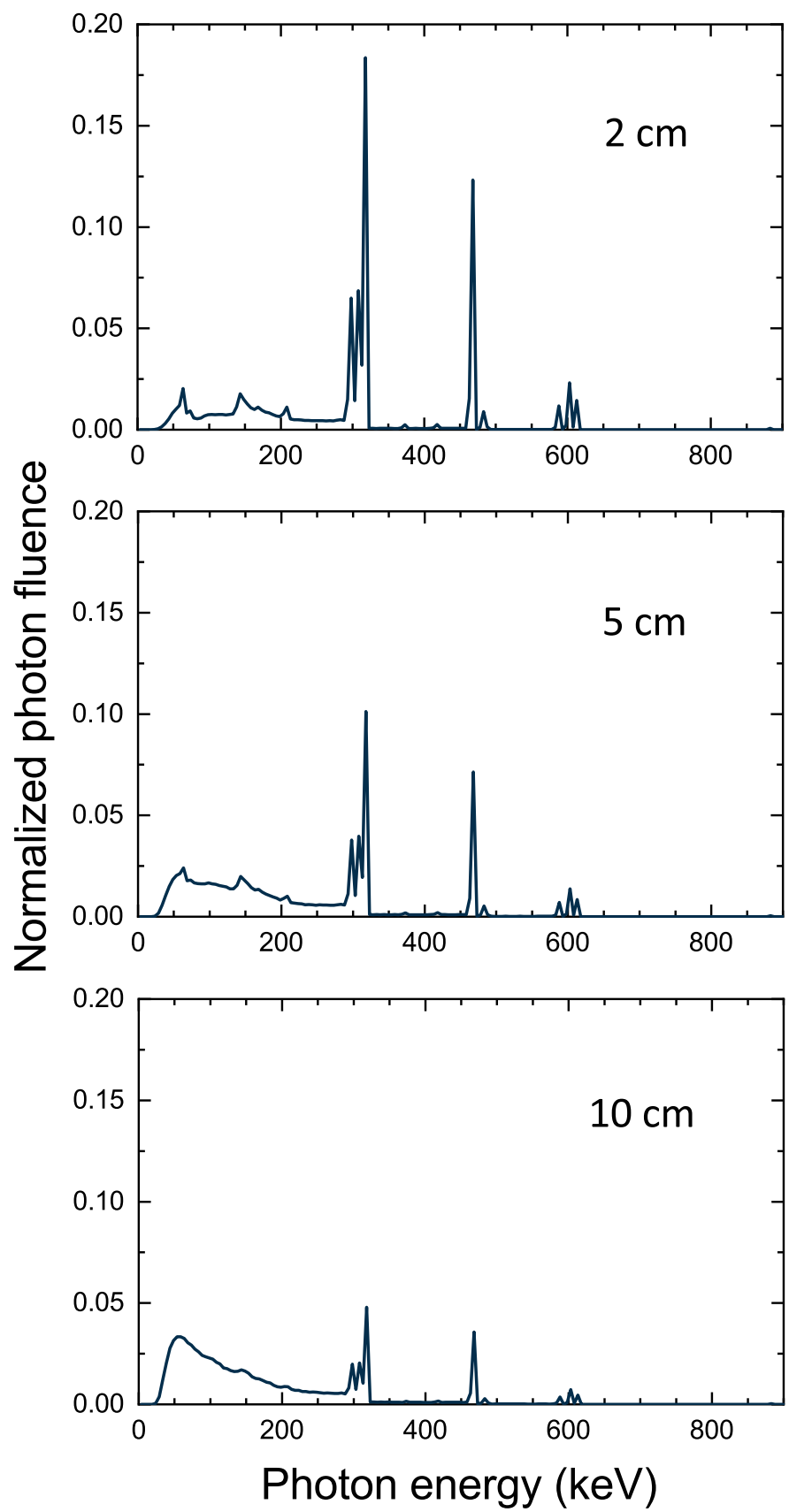

Figure 2.3: Photon fluence spectra differential in energy normalized to the total fluence for a given case in a small volume of water in a $40 \times 40 \times 40 \mathrm{~cm}^{3}$ water phantom at 2,5 , and $10 \mathrm{~cm}$ distances from ${ }^{192} \mathrm{Ir}$ source. The spectra were obtained with PENELOPE; $x$ and $y$ axes legend applies to all plots. 
dependence of the detector also changes with the source-to-detector distance, and it must be accounted for separately.

\section{Active volume size}

Though the end quantity of interest is absorbed dose to water at a point, it is not achievable in practice due to finite size of detector cavity. If the volume is not sufficiently small, volume averaging effect leads to underestimation of absorbed dose to water due to steep dose gradients around BT sources.

\section{Angular dependence}

Source reference dosimetry dataset determination, as well as in vivo dosimetry, include measurements where the polar angle $\theta$ between the source and the detector is not $90^{\circ}$. This may introduce angular dependence of the detector signal due to detector design features (e.g., if encapsulation is not axially symmetric). Another example could be the stem-effect in scintillator dosimetry where Cerenkov radiation is induced in the fiber cable. The effect becomes angle-dependent if the source is stepped in a needle alongside the detector. Important to note that the angular dependence should be distinguished from the volume averaging effect which can also depend on the irradiation angle if the detector active volume is elongated.

\section{Detector overview}

Previous section showed that an ideal detector should possess a number of properties, but we shall see here that they do not always go hand in hand. Despite that, lack of some properties does not necessarily imply lower accuracy in BT dosimetry measurements provided that the detector is well-characterized using MC and experimental methods, and that the applied correction factors do not introduce large uncertainties. The most important is that a compromise must be made considering the purpose of detector use.

For the determination of ${ }^{192} \mathrm{Ir}$ source reference datasets, passive readout detectors are not disadvantageous if they are water-equivalent and have other suitable properties to ensure high accuracy of treatment planning. But to facilitate dosimetry measurements for regular QA of treatment units at clinical sites, direct readout detectors would be preferrable. Detectors for real-time in vivo dosimetry of remote afterloading BT treatments must have high sensitivity and small volume to fit into a treatment needle; a combination of properties that is most easily achieved using medium- or high- $Z$ detectors.

There has been a number of detectors used or investigated for BT dosimetry. Some were passive readout detectors, such as TLDs, electron paramagnetic resonance detectors (e.g., alanine, lithium formate), gel dosimeters, radiochromic film. Others have direct readout: metal oxide semiconductor field effect transistors (MOSFETs), silicon diodes, diamond detectors, organic and 
Table 2.1: Mean atomic number $\bar{Z}$, mass density $\rho$, and electron density $n_{\mathrm{e}}$ for water and the selected detector media typically used in BT dosimetry and in this thesis. $\bar{Z}$ values for compounds were calculated using the Bragg additivity rule.

\begin{tabular}{lccc}
\hline Medium & $\bar{Z}$ & $\rho\left(\mathrm{g} \mathrm{cm}^{-3}\right)$ & $n_{\mathrm{e}}\left(\times 10^{23}\right.$ electrons cm $\left.^{-3}\right)$ \\
\hline Water & 6.6 & 0.998 & 3.337 \\
Polystyrene & 5.3 & 1.060 & 3.432 \\
Diamond & 6 & 3.515 & 10.574 \\
Alanine & 6.1 & 1.420 & 4.607 \\
Lithium formate & 6.7 & 1.480 & 4.585 \\
Lithium fluoride & 7.5 & 2.635 & 7.341 \\
$\mathrm{Al}_{2} \mathrm{O}_{3}$ & 11 & 3.970 & 11.724 \\
Silicon & 14 & 2.330 & 6.994 \\
$\mathrm{ZnSe}$ & 32 & 5.270 & 14.112 \\
CsI & 54 & 4.510 & 11.291 \\
\hline
\end{tabular}

inorganic scintillators. An extensive overview of detectors and their properties can be found in (Andreo et al 2017). Here, we describe TLDs to showcase why the most investigated and widely used dosimeters still leave room for other detectors for in-phantom QA of treatment units and in vivo dosimetry. Afterwards, we focus on diamond detectors and inorganic scintillators, both of which were the objects of Papers I-III and Paper IV, respectively. For comparison, atomic number and mass density of selected detectors are listed in Table 2.1. When the absorbed-dose energy dependence is discussed, one can also recall Figures 2.1 and 2.2 with the mass electronic stopping power and mass energy-absorption ratios of water to detector medium.

\section{Thermoluminescence dosimeters}

TLDs are considered a gold standard for the determination of source reference datasets for low-energy sources and recommended by the TG-43 formalism. There are no recommendations for high-energy sources, but TLDs are often the first-choice dosimeters (Pérez-Calatayud et al 2012). The main reason is their water-equivalence, relatively high sensitivity, and negligible dose rate dependence. TLDs operate as passive readout detectors where ionizing radiation creates electron-hole pairs that are trapped until dosimeters are heated. The resulting light yield is the detector readout signal. Typical pellet sizes are 1-4 $\mathrm{mm}$ in diameter and around $1 \mathrm{~mm}$ thickness (rectangular crystals have similar sizes). These dimensions and the range of secondary electrons created by ${ }^{192}$ Ir photon interactions in the detector cavity fulfill the large cavity theory conditions. As a result, the absorbed-dose energy dependence of TLDs can be evaluated using $\mu_{\mathrm{en}} / \rho$ ratios of water to detector medium which facilitates detector characterization. 
However, TLDs have non-negligible intrinsic energy dependence $R_{Q_{0}, Q}$ when they are calibrated in terms of absorbed dose to water in high-energy photon beams ( ${ }^{60} \mathrm{Co}$ and $\mathrm{MV}$ photon beams). Due to differences in the ionization track density of secondary electrons, the light yield per absorbed dose to detector is up to $10 \%$ greater in the photon energy range covered by ${ }^{192} \mathrm{Ir}$ gamma emissions compared to that in the calibration beam quality (Davis et al 2003, Nunn et al 2008, Carlsson Tedgren et al 2011). Though the overall trend of over-response was consistent among the studies, its magnitude varied within several percent. The differences were attributed to the TLD composition and preparation (Olko et al 2002), pre- and post-annealing conditions (Carlsson Tedgren et al 2011). A failure to account for such dependence would result in systematic errors, but a single correction factor has not been proposed. Instead, it is advised to assign a $6 \%(k=2)$ uncertainty to the overall energy response correction factor when an extensive study cannot be performed (Pérez-Calatayud et al 2012). If $R_{Q_{0}, Q}$ is accounted for, the total uncertainty in $D_{\text {wat }}$ is reduced to $3-6 \%(k=2)$ (Raffi et al 2010). While careful determination of the absorbed-dose energy and the intrinsic energy dependences is feasible for obtaining high-accuracy source reference datasets, the detectors are not practical for regular in-phantom QA of treatment units at clinical sites and cannot be used for real-time in vivo dosimetry.

\section{Natural diamond detectors}

Natural diamond detectors operate as semiconductors where ionizing radiation creates electron-hole pairs that are collected at the electrodes. The material has the best combination of properties compared to other direct readout solid-state detectors: water-equivalence, high mass density, high resistance to radiation damage, low leakage current due to large bandgap of $5.5 \mathrm{eV}$, high electron and hole mobility (Canali et al 1978).

Nevertheless, their performance is compromised by impurities and crystal defects. In pure crystals, electron-hole recombination is directly proportional to the square root of their production, which, in turn, is governed by the dose rate (Fowler 1966). The amount of impurities must be balanced. If it is insufficient, recombination will dominate and will lead to a decrease in the collected charge. If it is too large, the same will occur because electrons will be trapped at recombination centers instead of reaching the electrodes. The control of crystal quality is not easily achieved for natural diamonds, and they exhibit a non-linear dose-rate dependence (Westermark et al 2000). A correction factor, called the recombination parameter, must be determined empirically. Once it is obtained, natural diamond detectors could be suitable for relative dosimetry of HDR ${ }^{192}$ Ir BT sources (Rustgi 1998, Laub 2002).

However, their use remained cumbersome because the recombination parameter is detector dependent (Laub et al 1997). Additionally, there is dependence on the accumulated dose until the traps created by impurities are filled 
with electrons, and the internal electric field in the crystal is saturated (Kozlov et al 1975). The induced polarization effect requires detector pre-irradiation before daily use and depends on not only the amount of impurities but also on the applied voltage. Due to detector individuality, the pre-irradiation dose can vary from 1 to 10 Gy (De Angelis et al 2002), and the reproducibility of measurements is difficult to ensure. Eventually, suboptimal performance combined with the scarcity of detector-grade quality crystals ceased the detector use.

\section{Synthetic diamond detectors}

With the advent of chemical vapor deposition (CVD) technique it became possible to grow high-quality synthetic diamond layers. They do not differ from natural ones in terms of the mass density, charge carrier mobility, atomic number, and crystal lattice structure. A distinct and highly advantageous difference is better control of impurities and crystal homogeneity using the CVD technique. As a result, the drawbacks limiting natural diamond detector application in radiotherapy dosimetry could be minimized or even eliminated.

Further advancements in synthetic diamond manufacturing technologies and increased interest in small-field high-energy photon beam dosimetry (Alfonso et al 2008), drove the development of synthetic diamond detectors for use in radiotherapy. The initial attempts were not satisfactory due to detector design and crystal quality issues (De Angelis et al 2007, Górka et al 2008), but eventually a single crystal synthetic diamond grown using the CVD technique was used to construct a detector that is now commercially available as microDiamond (PTW 60019, Freiburg, Germany). The nominal active volume of the detector is a cylinder with 1 micrometer thickness and $2.2 \mathrm{~mm}$ diameter. The detector works as a Schottky diode without external bias owing to the electrode-diamond junction that creates the built-in potential. More details on the construction and electric properties of the detector can be found in publications on its prototype (Almaviva et al 2008, Almaviva et al 2010).

The detector exhibited excellent performance in small-field high-energy external photon beam dosimetry: linear response to absorbed dose, negligible dose-rate dependence, high signal stability, low pre-irradiation dose, nearly negligible angular dependence, and no volume averaging effects (Ciancaglioni et al 2012, Laub and Crilly 2014, Lárraga-Gutiérrez et al 2015, Benmakhlouf et al 2015). These findings, combined with the detector characteristics, such as small volume, high water-equivalence, and direct readout, motivated to investigate the suitability of the microDiamond detector for dosimetry of HDR ${ }^{192}$ Ir BT treatment units. To our knowledge, there have been no publications on the detector use either for BT or kilovoltage photon beam dosimetry prior to Paper I. 


\section{Fiber-coupled inorganic scintillators}

The most suitable application type for inorganic scintillators is real-time in vivo dosimetry. Their high atomic number and mass density enable construction of dosimeters that have high sensitivity and fit into treatment needles (diameter less than $2.0 \mathrm{~mm}$ ). Despite that, inorganic scintillators have been under the radar while organic scintillators dominated the field due to their water-equivalence (Beaulieu and Beddar 2016). However, the organic scintillator signal is not high enough for accurate measurements farther than $7 \mathrm{~cm}$ from ${ }^{192}$ Ir sources (Lambert et al 2006, Linares Rosales et al 2019). A wide source-to-detector distance range is relevant for in vivo dosimetry where the detector is usually at the center of the tumor, and some source dwell positions are in the periphery. Additionally, the stem-effect is non-negligible, and though corrections are feasible, they are not trivial (Therriault-Proulx et al 2011, Archambault et al 2012). For high- $Z$ inorganic scintillators, on the other hand, the stem-effect correction is not needed due to high signal from the active volume, and they have been investigated with increasing interest (Kertzscher and Beddar 2019, Linares Rosales et al 2020b, Debnath et al 2021, Jørgensen et al 2021a).

There is a number of high- $Z$ inorganic scintillators and the choice depends on luminescence properties, such as scintillation stability, afterglow and memory effects, crystal transparency to its own light emissions, temperature dependence, as well as signal intensity compared with the stem-effect. ZnSe has been shown to have all suitable properties (Kertzscher and Beddar 2019) and is already tested for in vivo dosimetry (Debnath et al 2021, Jørgensen et al 2021a). Another high- $Z$ scintillator CsI is used in combination with organic scintillators (Linares Rosales et al 2020b). The most experimentally investigated inorganic scintillator is medium- $Z \mathrm{Al}_{2} \mathrm{O}_{3}$ (e.g., Andersen et al 2009, Johansen et al 2018). Therefore, these three detectors were selected for the MC-based detector characterization in terms of the absorbed-dose energy dependence in Paper IV. 


\section{Detector calibration and characterization}

In this chapter we address methods for detector calibration and characterization used in BT dosimetry. A general dosimetry formalism, which was applied in Paper I and Paper II, is presented in Section 3.1. Due to the lack of coherent guidelines in BT dosimetry, several pathways of detector calibration have evolved in the community and in Section 3.2 we discuss the two main choices. One of them is questioned in Paper IV regarding in vivo detector characterization. Section 3.3 introduces MC simulations of ionizing radiation transport used for detector characterization in this thesis.

\subsection{Determination of absorbed dose to water}

Due to the absence of regular in-phantom QA of treatment units and in vivo dosimetry using probe-type detectors, the need for BT dosimetry in terms of absorbed dose to water has been restricted to the determination of the doserate constant $\Lambda$ for source reference dosimetry datasets. Limited applications did not necessitate establishment of codes of practice which is in stark contrast to high-energy photon and electron EBRT dosimetry (Almond et al 1991, Andreo et al 2000).

However, similar principles apply, and we can draw a parallel with the EBRT codes of practice by defining detector calibration coefficient in terms of absorbed dose to water in beam quality $Q_{0}$ as follows

$$
N_{D, \mathrm{w}, Q_{0}}=\left[\frac{D_{\mathrm{wat}}}{M}\right]_{Q_{0}},
$$


where $D_{\text {wat }}$ is absorbed dose to water at a point of interest in the absence of detector cavity, and $M$ is detector readout corrected for influence quantities (temperature, pressure, and other not related to beam quality).

Subsequently, absorbed dose to water in the measurement beam quality $Q$ can be determined as follows

$$
D_{\text {wat }, Q}=M_{Q} N_{D, \mathrm{w}, Q_{0}} k_{Q, Q_{0}},
$$

where $M$ is detector readout corrected for influence quantities in the measurement beam quality $Q$, and $k_{Q, Q_{0}}$ is a beam quality correction factor. The beam quality correction factor $k_{Q, Q_{0}}$ accounts for the difference in detector response between the two beam qualities. It can be derived combining equations (3.1) and (3.2) as follows

$$
k_{Q, Q_{0}}=\frac{N_{D, \mathrm{w}, Q}}{N_{D, \mathrm{w}, Q_{0}}}=\frac{\left[D_{\mathrm{wat}} / M\right]_{Q}}{\left[D_{\mathrm{wat}} / M\right]_{Q_{0}}} .
$$

Detector calibration coefficients in equation (3.1) can be further rewritten in terms of two components and equation (3.3) becomes

$$
k_{Q, Q_{0}}=\frac{\left[D_{\mathrm{wat}} / \bar{D}_{\mathrm{det}}\right]_{Q}}{\left[D_{\mathrm{wat}} / \bar{D}_{\mathrm{det}}\right]_{Q_{0}}} \cdot \frac{\left[M / \bar{D}_{\mathrm{det}}\right]_{Q_{0}}}{\left[M / \bar{D}_{\mathrm{det}}\right]_{Q}}=f_{Q, Q_{0}} \cdot R_{Q_{0}, Q},
$$

where $\bar{D}_{\text {det }}$ is the average absorbed dose in the detector medium at the point of interest in beam quality $Q$ or $Q_{0}$. Quantity $D_{\text {wat }} / \bar{D}_{\text {det }}$ in a given beam quality is the absorbed-dose energy dependence of detector relative to water. The ratio can also be recognized from equations (2.4) and (2.5) concerning the cavity theory. The quantity $f_{Q, Q_{0}}$ describes water-equivalence of the detector cavity in a given beam quality and how it changes between the measurement and calibration beam qualities. It can be calculated using either cavity theory or MC simulations. If MC simulations are used, the quantity may also include corrections for volume averaging and fluence perturbation due to the extracameral components or the detector cavity itself.

The quantity $R_{Q_{0}, Q}$ is the relative intrinsic energy response of the detector. It quantifies the detector signal yield per average absorbed dose to the detector in a given beam quality and how it changes between the measurement and calibration beam qualities. Since general-purpose MC codes of ionizing radiation transport do not simulate signal generation in detectors, the determination of $R_{Q_{0}}$ relies on experimental methods. When detectors are calibrated in beam quality $Q_{0}$, the intrinsic energy response is calculated as follows

$$
R_{Q_{0}}=\left[\frac{M}{\bar{D}_{\mathrm{det}}}\right]_{Q_{0}}=\frac{1}{N_{D, \mathrm{w}, Q}} \cdot\left[\frac{D_{\mathrm{wat}}}{\bar{D}_{\mathrm{det}}}\right]_{Q_{0}}=\left[\frac{M}{D_{\mathrm{wat}}}\right]_{Q_{0}} \cdot\left[\frac{D_{\mathrm{wat}}}{\bar{D}_{\mathrm{det}}}\right]_{Q_{0}},
$$

where all quantities are the same as defined previously. We observe that the intrinsic energy response of the detector is the remaining part of the calibration coefficient after the absorbed-dose energy dependence of the detector 
relative to water is accounted for. The relative intrinsic energy dependence $R_{Q_{0}, Q}$ is obtained by taking a ratio of corresponding quantities.

A note must be made regarding the beam quality specification. In EBRT, the user's beam quality $Q$ is specified and determined under certain conditions (e.g., for high-energy photon beams, the tissue-to-phantom ratio $\mathrm{TPR}_{20,10}$ in the TRS-398 protocol or the percentage depth dose curve at $10 \mathrm{~cm}$ depth in water $\% d d(10)_{\mathrm{x}}$ in the TG-51 protocol). In BT, a strict beam quality specification does not exist. It has been proposed to use a ratio of absorbed dose to water values at 2 and $1 \mathrm{~cm}$ distances for a specific source type as the beam quality specification and to treat $k_{Q, Q_{0}}$ as a function of source-todetector distance (Quast et al 2016). The suggested notation has not caught on in the BT field. Instead, it is usually considered that the beam quality $Q$ changes with source-to-detector distance (namely, photon energy spectrum) with an implicit assumption of $k_{Q, Q_{0}}$ being a function of source-to-detector distance without making any distinction in the notation. This approach is also adopted in the thesis summary and Papers I-IV.

\subsection{Detector calibration}

Naturally, the lack of BT dosimetry codes of practice resulted in inconsistent calibration methods of probe-type detectors. The only recommendation on that matter is that the calibration beam quality should resemble the measurement beam quality, and if not possible, any differences in detector response between the two beam qualities should be accounted for (Rivard et al 2004, Pérez-Calatayud et al 2012).

There are two main pathways of detector calibration adopted in BT dosimetry:

1. With traceability to primary standards of high-energy external photon beam radiotherapy in terms of absorbed dose to water.

2. With traceability to primary standards of air-kerma strength of ${ }^{192} \mathrm{Ir}$ source using a clinical source and TPS-calculated absorbed dose to water.

\section{Traceability to primary standards of high-energy external photon beam radiotherapy}

Recalling the discussion in Section 2.2, there are developments of absorbed dose to water primary standards for ${ }^{192} \mathrm{Ir}$ sources, but they are for source calibration and dosimetry. It is not likely that the efforts will result in probetype detector calibration using such standards. Therefore, detector calibration in terms of absorbed dose to water must be done using primary standards for ${ }^{60} \mathrm{Co}$ and $\mathrm{MV}$ external photon beams. We adopted this practice 
in Paper I and calibrated detectors in terms of absorbed dose to water in ${ }^{60}$ Co beam following the TRS-398 code of practice at the Swedish secondary standards dosimetry laboratory. The chosen calibration method offered low uncertainty in the calibration coefficient, $u\left(N_{D, \mathrm{w}, Q_{0}}\right)=1.0 \%(k=2)$, and a TPS-independent determination of absorbed dose to water around HDR ${ }^{192} \mathrm{Ir}$ sources. The approach has been used for electron paramagnetic resonance dosimeters (Antonovic et al 2009, Anton et al 2015), TLDs and radiochromic film (Pérez-Calatyud et al 2012 and references therein), but considered prone to systematic errors unless the relative intrinsic energy dependence $R_{Q_{0}, Q}$ of detectors is determined alongside.

\section{Traceability to primary standards of air-kerma using clinical sources}

Following this method, detectors are calibrated at a chosen reference point in water or water-equivalent plastic phantoms using clinical ${ }^{192} \mathrm{Ir}$ sources. In this case, $D_{\text {wat }}$ in equation (3.1) is replaced with $D_{\text {wat,TG43 }}$ which is the absorbed dose to water at a point of interest calculated using the TG- 43 formalism. The method is most often used for in vivo detector calibration (e.g., Andersen et al 2009, Kertzscher and Beddar 2019, Linares Rosales et al 2020a, Ruiz-Arrebola et al 2020, Debnath et al 2021, Jørgensen et al 2021a). The measurement and calibration beam qualities remain essentially the same compared to the first method. It may appear as an advantage since detector characterization in terms of the absorbed-dose energy and intrinsic energy dependences covers a narrower beam quality range.

On the other hand, the method does not allow for independent determination of the absorbed dose to water. An implicit assumption is that the source reference data in a TPS is correct and that no errors occur in source positioning and timing during detector calibration. That is, some of the most important treatment delivery aspects that a high-accuracy in vivo dosimetry system is aiming to detect (Kertzscher et al 2014b) would likely be missed because they would be conflated with the detector calibration coefficient.

Another major problem is positioning of the detector itself. A reference position for detector calibration is usually chosen at around 1-2 cm sourceto-detector distance as a compromise between uncertainties related to detector positioning and signal strength. Given a rigid setup and well-controlled measurement conditions, detector positioning uncertainty of $0.2-0.5 \mathrm{~mm}$ on the transversal place can be achieved, but due to steep dose gradients close to ${ }^{192}$ Ir sources, it would still translate to the uncertainty of around $4 \%(k=2)$ in the absorbed dose to water (Andersen et al 2009, Ruiz-Arrebola et al 2020). If there is a discrepancy between the experimentally determined and TPScalculated absorbed dose to water values, it is disguised in the detector positioning uncertainty. Once again, it is not possible to distinguish whether 
there is an error in treatment delivery or in detector positioning during the calibration.

The uncertainty of the source air-kerma strength $S_{K}$ or RAKR further contributes to the total uncertainty budget of the detector calibration coefficient. Source calibration using direct methods for source standard realization has the total uncertainty of $0.7 \%(k=2)$ (Sander and Nutbrown 2006), whereas calibration using the interim standards results in the uncertainty of approximately 3\% $(k=2)$ (Soares et al 2009, DeWerd et al 2011). Other uncertainties related to $D_{\text {wat,TG43 }}$ calculations are not straightforward to estimate but could amount to several percent as discussed in Section 2.2 regarding the source reference dataset dosimetry. For instance, Ruiz-Arrebola et al (2020) estimated a total uncertainty of nearly $10 \%(k=2)$ in the detector calibration coefficient for MOSFET in vivo detectors. The value is substantially greater than the one employing the first calibration method in terms of $N_{D, \mathrm{w}, Q_{0}}$.

Detector calibration method using clinical sources and its implications on in vivo detector characterization and dosimetry accuracy are further questioned in Paper IV. There we also address how phantom size can affect detector calibration in small water-equivalent phantoms that are employed for convenience (Kertzscher et al 2014a, Jørgensen et al 2021b). In-phantom scatter conditions are not the same in patients and the determined calibration coefficient may not be valid.

Here, it is interesting to notice an indirect connection with the study on synthetic diamond detector in Paper I and Paper II. Given a high-accuracy and convenient to use detector, regular end-to-end in-phantom QA of treatment units could be performed. This would ensure that the treatment units perform as expected, and if chosen, the method of detector calibration using clinical sources would be more reliable.

\subsection{Monte Carlo simulations}

From the discussion on cavity theory, it is evident that detector characterization in terms of the absorbed-dose energy dependence relative to water plays a key role in experimental dosimetry. While cavity theory provides fundamental understanding of what detector medium shall be chosen considering beam quality and cavity dimensions, MC simulations of ionizing radiation transport became a primary choice for detector characterization in radiotherapy (Andreo 2018). Adhering to the general formalism presented in Section 3.1, the change in the absorbed-dose energy dependence $f_{Q, Q_{0}}$ must be determined. There, MC simulations offer higher accuracy than experimentally determined values. The method allows, but is not restricted to, simulating energy deposition in the medium of interest, scoring particle fluence, investigating volume averaging effects and particle fluence perturbation by extracameral components of the detector. All these aspects were studied for synthetic diamond 
detectors in Paper I and Paper II, and for inorganic scintillators in Paper IV to a varying degree.

In this thesis work, we used a general-purpose MC code system PENELOPE (Salvat 2019) with the main program penEasy (Sempau et al 2011) for scoring tallies of interest, and a subroutine package PENGEOM to define bodies for detectors and irradiation setups. PENELOPE simulates electron, positron and photon transport in the energy range from $50 \mathrm{eV}$ to $1 \mathrm{GeV}$ (giving qualitative results below $1 \mathrm{keV}$ energy). The code implements detailed photon transport and mixed (class II) transport for electrons and positrons. It is well-benchmarked and, together with another MC code system EGSnrc (Kawrakow et al 2013), is the most used for dosimetry in radiotherapy. A description of physics underlying radiation transport simulations and the inner workings of PENELOPE is found in the manual. A comprehensive review of MC simulation use for radiotherapy dosimetry can be found in (Andreo 2018).

There is a continuous improvement of ionizing radiation transport modelling, as well as Type B evaluation of uncertainties associated with lowenergy photon interaction cross sections and their implementation in MC codes (Valdes-Cortez et al 2021 and references therein). Concerns regarding modelling of sub-kilovoltage kinetic energy electrons have also been addressed (Thomson and Kawrakow 2011). Understanding approximations underlying the physics implemented in the code and cross section data uncertainties is crucial for result evaluation. But it is no less important to keep in mind fundamental limitations once MC simulations are used. That is, which aspects of detector response they model and which parameters are under user's control. It is a recurrent theme in Papers I-IV that there is a chain of events from energy deposition to signal collection, and MC simulations of ionizing radiation transport model only the first step. The thesis was done with the awareness to that, especially given that the investigated synthetic diamond detectors are not well-researched and non-dosimetric properties play a role as addressed in Paper I and Paper II. The same applies to inorganic scintillators, some of which were studied in Paper IV. Inorganic scintillator response can be affected by luminescence properties (Kertzscher and Beddar 2017, 2019), and one must strongly consider which detectors shall be chosen for investigation using MC simulations. Otherwise, decoupling of the MC-calculated absorbeddose energy dependence and experimentally determined detector properties is complicated and no meaningful conclusions can be drawn.

Finally, all simulations further depend on user-controlled geometry input files, radiation transport parameters, and material definition. Geometry definition is rather straightforward, and a set of radiation transport parameters can be selected according to the simulated beam quality and scoring volume sizes. Specification of material properties, on the other hand, can be ambiguous depending on the case. The issue is brought up for the diamond medium in Paper III. Diamond is often assumed to be graphite-like, but it is not 
correct as highlighted by Fernández-Varea et al (2021). Such an assumption can have consequences for MC simulation results, as well as for the calculation of $S_{\mathrm{el}} / \rho$ values using MC codes or the relativistic Bethe formula (ICRU 90). 



\section{Contributions of our research}

\subsection{Synthetic diamond detector for dosimetry in ${ }^{192} \mathrm{Ir}$ brachytherapy}

Inferring from the synthetic diamond detector microDiamond (PTW 60019) properties and performance in small-field high-energy EBRT dosimetry discussed in Section 2.4, in Paper I we raised a hypothesis that its use could be extended to in-phantom QA of HDR ${ }^{192} \mathrm{Ir}$ BT remote afterloading units in terms of absorbed dose to water. To strengthen the work, we used three microDiamond detectors because some studies indicated inter-detector variability in pre-irradiation dose, as well as in over-response in small-field high-energy photon and proton beams. Our measurements were done in a $20 \times 20 \times 20 \mathrm{~cm}^{3}$ polymethyl methacrylate phantom at $1.5,2.5$, and $5.5 \mathrm{~cm}$ source-to-detector distances using two HDR ${ }^{192}$ Ir BT treatment units from different vendors.

Given shortcomings of natural diamond detectors in BT dosimetry and no published studies on the microDiamond detector in low-energy photon beams, it was important first to examine short- and long-term stability, and the dose-rate dependence of the detectors. The investigation was done in ${ }^{60} \mathrm{Co}$ beam under well-controlled measurement conditions. Pre-irradiation with less than 2 Gy was sufficient to stabilize detector signal, and there was no substantial difference among the samples. The recombination parameter determined for one of the detectors was $1.001 \pm 0.003(k=2)$ in the dose-rate range from 0.3 to $2.8 \mathrm{~Gy} \mathrm{~min}^{-1}$, i.e., the dose-rate dependence was negligible. Based on an extensive literature study on diamond detectors, that can be found in Paper I, the short-term stability results implied that the dose-rate 
dependence should be comparable for all detectors. Hence, no correction factors were applied to the detector signal in ${ }^{192} \mathrm{Ir}$ beams.

Beam quality correction factors $k_{Q, Q_{0}}$ were calculated using the PENELOPE MC code. The values accounted only for the absorbed-dose energy dependence $f_{Q, Q_{0}}$ between the measurement beam ${ }^{192} \mathrm{Ir}$ and the calibration beam ${ }^{60}$ Co. The relative intrinsic energy dependence $R_{Q_{0}, Q}$ was assumed to be unity. The values for $k_{Q, Q_{0}}$ varied within $2 \%$ over the investigated source-to-detector distance range. Experimentally determined absorbed dose to water values (see equation (3.2)) agreed with the TPS-calculated values within $3 \%$. Experimental uncertainties were from 2 to $4 \%(k=2)$ depending on the source-to-detector distance. The agreement and experimental uncertainties were comparable to or better than for passive readout detectors used in analogous studies (Antonovic et al 2009, Raffi et al 2010, Carlsson Tedgren et al 2012, Anton et al 2015) and superior to direct readout detectors, such as plastic scintillators (Lambert et al 2006) and MOSFETs, that have greater absorbed-dose energy dependence, high inter-detector variability and dependence on the accumulated dose (Zilio et al 2006, Qi et al 2007). The findings strongly suggest that synthetic diamond detectors could be adopted for high-accuracy in-phantom QA of ${ }^{192}$ Ir BT treatment units. Additionally, the results of our study prompted investigations of detector use for HDR ${ }^{192} \mathrm{Ir}$ source reference dataset dosimetry (Rossi et al 2019, Rossi et al 2020) motivated by the need to validate the MC-calculated values as discussed in Section 2.2. Their findings agreed with ours in terms of the absorbed-dose energy and dose-rate dependences of the detectors.

However, detector-to-detector variation may require individual characterization, or alternatively, Type B evaluation of uncertainties due to detector construction. Though well within expanded uncertainties, experimentally determined absorbed dose to water values were systematically higher for one of the synthetic diamond detectors in our study. Similar differences were observed in other studies (Rossi et al 2019, Rossi et al 2020). Additionally, the detector has complicated inner structure with metallic pins. An x-ray image is shown in a study by Andreo et al (2016) where they raised an additional question regarding the active volume dimensions provided by the manufacturer. Marinelli et al (2016) showed that the actual dimensions were close to the nominal ones, but the discussion encouraged the manufacturer to update blueprints by including all metallic structures (the supporting pins and contacts). It did not affect MC-calculated small-field correction factors (Hartmann and Zink 2019), but had implications for our study. The $k_{Q, Q_{0}}$ values given in Paper I were calculated using complete blueprints. When the metallic structures were not accounted for, the $k_{Q, Q_{0}}$ values were greater by 1 to $3 \%$ for 1.5 to $5.5 \mathrm{~cm}$ source-to-detector distances. The change is not negligible given a few percent variation over the entire range and statistical uncertainties below $1 \%(k=2)$. Such issues could be avoided by constructing 
synthetic diamond detectors with more water-equivalent extracameral components (Marsolat et al 2015).

$\mathrm{MC}$ calculations for detector characterization did allow for introducing a new detector in BT dosimetry, but at the same time, adoption of such detectors comes with new challenges not encountered in BT where the use of large cavity theory has been prevalent due to the simplicity of typical detector design. It does not compromise the application of synthetic diamond detectors for in-phantom QA of BT treatment units, but limitations of the MC-calculated absorbed-dose energy correction factors have to be considered.

\subsection{Synthetic diamond response in kilovoltage photon beams}

In Paper I, we assumed that the relative intrinsic energy dependence $R_{Q_{0}, Q}$ of the synthetic diamond detector was unity between ${ }^{60} \mathrm{Co}$ and ${ }^{192} \mathrm{Ir}$ beam qualities. No systematic discrepancy between the TPS-calculated and experimentally determined $D_{\text {wat }}$ values supported the assumption, but it had to be verified. Therefore, the aim of Paper II was to investigate the synthetic diamond detector response in kilovoltage photon beams relative to the calibration beam ${ }^{60} \mathrm{Co}$. Additionally, we raised a hypothesis that the application of the detectors could be extended to low-energy photon beams, for instance, electronic BT sources.

We calibrated three microDiamond detectors in terms of air-kerma free in air $N_{K \text {,air }}$ in six CCRI x-ray beam qualities $(25-250 \mathrm{kV})$ at the Swedish secondary standards dosimetry laboratory. The intrinsic energy response $R_{Q}$ was extracted from detector calibration coefficients following the formalism in Section $3.1\left(N_{D, \mathrm{w}, Q_{0}}\right.$ was replaced with $N_{K \text {,air }}$ in equation $\left.(3.5)\right)$. The reference beam quality $Q_{0}$ was ${ }^{60} \mathrm{Co}$, and $R_{Q_{0}}$ was determined from detector calibration coefficients $N_{D, \mathrm{w}, Q_{0}}$ given in Paper $\mathbf{I}$. We found that the relative intrinsic energy dependence $R_{Q, Q_{0}}$ increases with the decreasing photon energy. Detector signal per average absorbed dose to the detector is 2.5 times greater in $50 \mathrm{kV}$ photon beam (effective photon energy $23 \mathrm{keV}$ ) compared to that in ${ }^{60} \mathrm{Co}$ beam. The $R_{Q, Q_{0}}$ values gradually dropped to 1.2 in $250 \mathrm{kV}$ beam (effective photon energy $127 \mathrm{keV}$ ) and most likely approach unity with the increasing photon energy. For comparison, the study included two unshielded silicon diodes (PTW 60017). Their relative intrinsic energy dependence $R_{Q, Q_{0}}$ varied from 0.97 to 1.09 , which is comparable to passive readout detectors discussed previously. A qualitative result evaluation using an ophthalmic ${ }^{125} \mathrm{I}$ BT seed (mean photon energy $28 \mathrm{keV}$ ) showed that $D_{\text {wat }}$ determined using synthetic diamond detectors would be over-estimated compared to the TPS-calculated values if the intrinsic energy dependence $R_{Q_{0}, Q}$ was not accounted for. 
Since the results rely on the MC-calculated absorbed-dose energy dependence $\left(D_{\text {wat }} / \bar{D}_{\text {det }}\right.$ and $\left.K_{\text {air }} / \bar{D}_{\text {det }}\right)$, accurate modelling of detector geometry and materials is essential. A nominal detector model is used in MC simulations, whereas as pointed out in Paper I, synthetic diamond detectors exhibit inter-detector variability. Calibration coefficients $N_{D, \mathrm{w}, Q_{0}}$ of synthetic diamond detectors used in Paper I and Paper II differed by 30\% and that could have been explained by differences in their active volume thickness (Marinelli et al 2016). However, calibration in terms of $N_{K \text {,air }}$ in Paper II revealed that there are other factors contributing to the detector response at low photon energies. Comparing the three synthetic diamond detectors, the

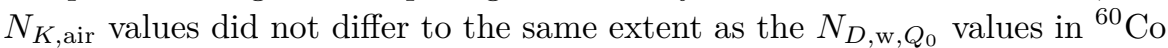
beam, and the difference was most pronounced at $25 \mathrm{kV}$ beam. The two silicon diodes used in our study did not exhibit such a large variation in $N_{K \text {,air }}$, and the existing one was explained by differences in their active volume size. Later, Khan et al (2020) reported similar findings for the synthetic diamond detector but did not proceed determining $R_{Q_{0}, Q}$.

We performed sensitivity analysis of detector blueprints by simulating thicker active volume $(2 \mu \mathrm{m}$ instead of $1 \mu \mathrm{m})$ and detectors with no metallic extracameral components. The resulting change in $R_{Q}$ was within $10 \%$ and decreased at higher $\mathrm{kV}$ energies. Therefore, it is most likely that the intrinsic energy dependence of diamond detectors is not a result of incomplete detector modelling, and it arises presumably due to electric or solid-state state physics effects in the detector. For instance, how charge collection is affected by conformity of the electric field, extension of the diffusion layer, charge imbalance in structural components, and other. Such occurrences have been observed by others and are discussed in detail in Paper II. Given that $R_{Q, Q_{0}}$ increases with the decreasing photon energy (i.e., increasing linear energy transfer of secondary electrons) it was not clear whether the same reasoning applies as for passive readout detectors. Additionally, inconclusive results were reached by others using synthetic diamond detectors in proton and heavier ion beams where the linear energy transfer is greater. Thus, the findings necessitate further investigation.

Paper II complements the synthetic diamond detector characterization presented in Paper I adhering to our chosen dosimetry formalism and TG-43 recommendations. The detector has substantial relative intrinsic energy dependence in $\mathrm{kV}$ photon beams relative to the calibration beam ${ }^{60} \mathrm{Co}$, but there are no indications that it would influence dosimetry of ${ }^{192} \mathrm{Ir}$ sources. Though there are photons with energies below $100 \mathrm{keV}$, the mean energy of ${ }^{192}$ Ir photon spectra is around $350 \mathrm{keV}$ in the investigated distance range. Non-relative dosimetry of electronic BT or low-energy photon-emitting sources could be subject to systematic errors. The values of $R_{Q, Q_{0}}$ were obtained for spectral photon beams, and further investigation is needed to evaluate how the results would apply to other beam qualities. On the other hand, relative dosimetry 
of $\mathrm{kV}$ photon beams and electronic BT sources was shown to be feasible by Damodar et al (2018) and Santiago Ayala Alvarez et al (2020), respectively.

\subsection{Diamond material properties for Monte Carlo simulations}

The results in Paper II forced to question what causes the intrinsic energy dependence of synthetic diamond detectors. As touched upon in Section 3.3, there are uncertainties associated with approximations and assumptions used for radiation transport modelling in $\mathrm{MC}$ codes. Experiments at low photon energies are complicated and done mainly for air, water, and graphite which are key dosimetric materials. Comparisons via the mass energy-absorption coefficients show an agreement within several percent for air (Büermann et al 2006). Evaluation of the state-of-the-art MC codes, among which the PENELOPE code, and various cross section databases also show an agreement within few percent in kilovoltage photon energy range for air, water, and graphite (Andreo et al 2012). A sensitivity analysis done in Paper II showed that a $2 \%$ increase in the photoelectric effect cross section in diamond resulted in a few percent change in the energy imparted. Thus, it could not account for the observed two-fold increase in $R_{Q, Q_{0}}$ at low photon energies. Experimental studies on electric and solid-state physics properties of the detector were beyond the scope of the thesis, and instead, we focused on material properties that affect $\mathrm{MC}$ simulations of ionizing radiation interactions in diamond.

The main user-selected material properties in the PENELOPE code are the atomic number $Z$, mass density $\rho$, mean excitation energy called the $I$-value, and number of conduction electrons per atom (here, denoted $n_{\text {ce }}$ ). The ICRU 90 Report (ICRU 90) provides key dosimetric data for graphite and other materials but not for diamond. Majority of studies on diamond detector response (including Paper I and Paper II) treat diamond as crystalline graphite with $I=81 \mathrm{eV}$ and $n_{\mathrm{ce}}=1$ but with higher density $\rho=3.515 \mathrm{~g} \mathrm{~cm}^{-3}$ (for crystalline graphite, $\rho=2.265 \mathrm{~g} \mathrm{~cm}^{-3}$ ). At first sight, it does not appear strange given that they both are carbon allotropes $(Z=6)$. However, Fernández-Varea et al (2021) pointed out that diamond has a different dielectric response function and is an insulator $\left(n_{\mathrm{ce}}=0\right)$, unlike conducting graphite. Subsequently, the $I$-value is $(88.5 \pm 2.0) \mathrm{eV}$ for diamond versus $(81.0 \pm 1.8) \mathrm{eV}$ for crystalline graphite.

A simplified expression of the relativistic Bethe formula illustrates how material properties $\left(\rho, I, n_{\text {ce }}\right)$ affect mass electronic stopping power of light charged particles traversing the medium

$$
\frac{S_{\mathrm{el}}(E)}{\rho} \propto \frac{Z}{A} \frac{1}{\beta^{2}}\left[\xi(\beta ; I)+F^{ \pm}(\beta)-\delta\left(\beta ; \rho, I, n_{\mathrm{ce}}\right)\right] .
$$

More details are in Paper III, but the important aspect here is that the $I$-value affects $S_{\text {el }} / \rho$ directly via the first term $\xi$ and also via the density- 
effect correction term $\delta$ as emphasized in (Andreo and Benmakhlouf 2017). We further clarified that the $\delta$-term depends on the number of conduction electrons per atom $n_{\text {ce }}$ which went unnoticed before. Considering how the change in the $I$-value affects detector dosimetry, it is worth recalling the role of cavity theory introduced in Section 2.3. Depending on the size of the diamond cavity and beam quality, an increase in the $I$-value would affect light charged particle energy deposition in cavities that can be approximated as small or intermediate, which was the case for the synthetic diamond detector in beam qualities described in Paper I and Paper II.

Therefore, we used the PENELOPE MC code to quantify the change in energy imparted in diamond medium if the new $I$-value of $88.5 \mathrm{eV}$ was adopted. We created cavities with different thickness from 1 to $30 \mu \mathrm{m}$ and $2.2 \mathrm{~mm}$ diameter to mimic the commercial synthetic diamond detector and explore hypothetical cases. We simulated a range of beam qualities where diamond detector has already been used or has potential: therapeutic x-rays $25-180 \mathrm{kV}$, photon-emitting BT sources $\left({ }^{125} \mathrm{I},{ }^{169} \mathrm{Yb},{ }^{192} \mathrm{Ir}\right), \beta$-source ${ }^{106} \mathrm{Ru}$, calibration beam ${ }^{60} \mathrm{Co}, 6 \mathrm{MV}$ linear accelerator beams (small and large fields), and veryhigh-energy $100 \mathrm{MeV}$ electron beam.

Depending on the beam quality and diamond cavity thickness, the change ranged from negligible to around $1.5 \%$ with statistical uncertainties less than or equal to $0.5 \%(k=2)$. Regarding implications for Paper I and Paper II results, the effect would be close to negligible. Quantities $f_{Q, Q_{0}}$ and $R_{Q_{0}, Q}$ involve ratios of the average absorbed dose to the detector cavity, and the change in the energy imparted was comparable for $1 \mu \mathrm{m}$ thickness diamond cavity in ${ }^{192} \mathrm{Ir}$, kilovoltage photon and ${ }^{60} \mathrm{Co}$ beams. However, it would be important for non-relative dosimetry or when electron spectra differ between the compared cases.

We further calculated the density-effect correction term for graphite, diamond and two pseudo-diamonds, that are simulated in most current studies. The difference among the $\delta$-terms was greatest at electron kinetic energies below $1 \mathrm{MeV}$ where the density-effect correction term contribution to $S_{\mathrm{el}} / \rho$ is less than 5\%. A non-negligible difference remained above $10 \mathrm{MeV}$ where the contribution cannot be neglected. The $\delta$-terms were calculated with Sternheimer's approach (ICRU 90 and references therein). There is also Fano's approach (Fano 1963), which is implemented in the PENELOPE code. The two methods handle the dielectric response and optical electron energy loss functions slightly differently, but comparison in our study showed that the results agree completely for diamond and graphite media at electron kinetic energies where the $\delta$-term is relevant. Another MC code EGSnrc allows using an auxiliary file with the pre-calculated $\delta$-term values or entering medium-dependent fitting parameters that generate such a function. Studies on diamond detectors with EGSnrc often employ the first option and compute the $\delta$-term values with the ESTAR program that implements Sternheimer's approach (Berger et al 2017). For custom materials, including diamond, a user can enter atomic 
composition, mass density $\rho$, and the $I$-value, but not $n_{\text {ce }}$. We observed that the program assumes $n_{\text {ce }}=2$ whenever $Z=6$, i.e., complying with the outdated recommendations for graphite (ICRU 1984). As a result, the generated density-effect correction term yields unphysical results for diamond and does not adhere to the new recommendations for graphite either. Therefore, in our study we provided the correct density-effect correction function for diamond calculated using Sternheimer's approach and the fitting parameters for such a function. Either could be chosen for diamond modelling in EGSnrs or for mass electronic stopping power calculations for more consistent results.

Finally, we emphasized that the same set of $\left(\rho, I, n_{\mathrm{ce}}\right)$ values should be chosen for MC simulations and for mass electronic stopping power calculations. There have been studies where different sets of values were used (e.g., Mobit et al 1997, Andreo and Benmakhlouf 2017, Paper II). It leads to inconsistent comparison of results. Such an approach may introduce systematic errors of several percent when $S_{\mathrm{el}} / \rho$ values are used either for theoretical discussions or for the estimation of absorbed dose to water using small or intermediate cavity theories.

Though far from explaining the results in Paper II, the study clarified which diamond material properties model the medium most faithfully and improved understanding of the energy deposition aspects in diamond for a range of beam qualities extending beyond BT dosimetry.

\subsection{Inorganic scintillators for in vivo dosimetry in ${ }^{192} \mathrm{Ir}$ brachytherapy}

The study object in Paper IV is high- $Z$ inorganic scintillators: ZnSe $(\bar{Z}=$ $\left.32, \rho=5.27 \mathrm{~g} \mathrm{~cm}^{-3}\right)$, CsI $\left(\bar{Z}=54, \rho=4.51 \mathrm{~g} \mathrm{~cm}^{-3}\right)$, and $\mathrm{Al}_{2} \mathrm{O}_{3}(\bar{Z}=11$, $\left.\rho=3.97 \mathrm{~g} \mathrm{~cm}^{-3}\right)$. The detector of interest was ZnSe while the other two were used for comparison when relevant. Unlike in Paper I and Paper II, the aim of the work was not to investigate detector suitability for BT dosimetry but rather to address how they are investigated given the in vivo dosimetry issues addressed in Chapters 2 and 3. We argue that the experimentally determined values of $D_{\text {wat,TG43 }} / M$ as a function of source-to-detector distance are not the same as the absorbed-dose energy dependence of the detector. The detector signal can be affected by dose-rate dependence, intrinsic energy dependence and other factors that will influence the determined function. Additionally, dose gradients are high and detector positioning uncertainties compromise the accuracy. Another issue specific to high- $Z$ detectors is the measurement conditions. The absorbed-dose energy correction function determined under full scatter conditions may not be the same under limited scatter conditions during in vivo measurements.

Therefore, we used the PENELOPE code to calculate the absorbed-dose energy dependence of detectors under conditions relevant for in vivo dosimetry 


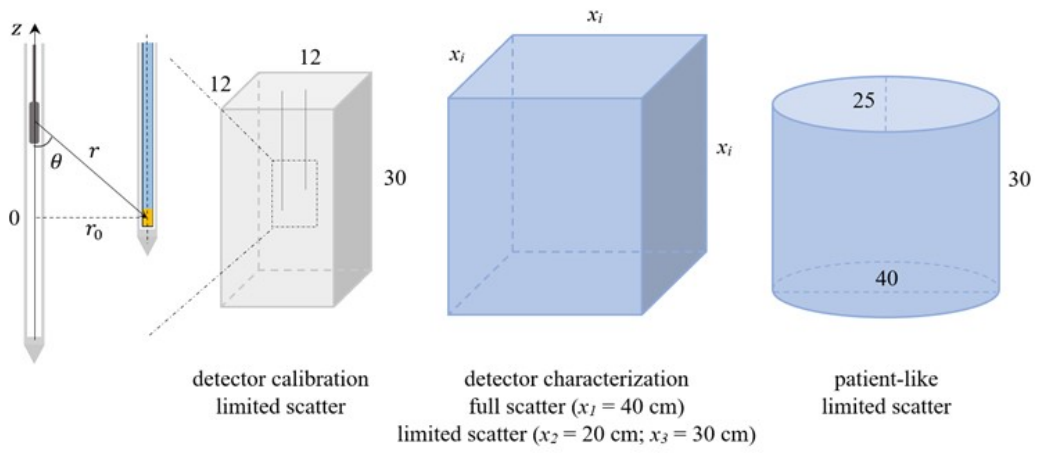

Figure 4.1: A sketch of source and detector needles describing terminology and phantoms used in Monte Carlo simulations (dimensions are in centimeters). The $z$ coordinate denotes position of the source with respect to the geometrical center of the active volume of a detector $(z=0), r$ is the radial distance from the center of the source to the geometrical center of the active volume of the detector, and $\theta$ is the polar angle between the longitudinal axis of the source and the geometrical center of the detector at distance $r$. When $\theta=90^{\circ}, r$ is denoted as $r_{0}$.

in ${ }^{192}$ Ir BT treatments. We focused on how the in-phantom scatter component affects detector response in several real-case measurement scenarios shown in Figure 4.1. Having in mind the most common prostate treatments, we further evaluated whether the presence of bones affects energy deposition in ZnSe. Since the source is stepped in a needle alongside the detector, volume averaging and detector design influence were also quantified.

Under full scatter conditions in a $40 \times 40 \times 40 \mathrm{~cm}^{3}$ water phantom, the absorbed-dose energy dependence of $\mathrm{ZnSe}$ and CsI was up to four and ten times higher, respectively, relative to water in the investigated range $r_{0}$ from 0.7 to $5.0 \mathrm{~cm}$. For $\mathrm{Al}_{2} \mathrm{O}_{3}$, the change was within $10 \%$ which is not unexpected given differences in $\bar{Z}$ among the three materials. An interesting finding there was that despite a much higher $\bar{Z}$ of CsI, its relative absorbed-dose energy dependence (normalized at $r_{0}=2 \mathrm{~cm}$ ) varied to the same extent as that for ZnSe. It is further explained in Paper IV based on the dominating photon interaction processes. High- $Z$ scintillators have not been explored extensively in BT dosimetry assuming their strong absorbed-dose energy dependence. The assumption is valid in absolute terms of the absorbed-dose energy dependence but not when the ratios are taken, i.e., equivalent to the $f_{Q, Q_{0}}$ quantity in equation (3.4). Thus, new high- $Z$ detectors having suitable luminescence properties could be introduced without increasing the absorbed-dose energy correction factors.

Addressing the experimental practice of detector characterization under full scatter conditions, we found that the absorbed-dose energy dependence of ZnSe does depend on the in-phantom scatter component. An additional correction of up to $10 \%$ is needed for in vivo measurements at $r_{0}$ distances beyond 
$2 \mathrm{~cm}$. It was not the case for $\mathrm{Al}_{2} \mathrm{O}_{3}$ which is more water-equivalent. Since neither detector response is affected by in-phantom scatter up to $r_{0}=2 \mathrm{~cm}$, pre-treatment constancy check and calibration of detectors in a limited scatter water-equivalent phantom could be performed as advised (Kertzscher et al 2014a, Jørgensen et al 2021b). The change in photon spectrum due to the presence of bones did not affect energy deposition in ZnSe for clinically relevant cases. Further simulations of hypothetical cases showed that it is the proximity to the bones that is important, thus the influence of prostate calcifications, which can surround treatment needles, remains to be investigated.

Finally, we presented a preliminary comparison of MC-calculated absorbed-dose energy dependence for ZnSe and corresponding experimental measurements for the same type of detector ( $\mathrm{ZnSe}: \mathrm{O})$ with a robotic arm for high-accuracy positioning (Jørgensen et al 2021a). The agreement was within expanded total uncertainties for two measurement scenarios, i.e., $\left(r_{0}\right.$ changes, $\left.\theta=90^{\circ}\right)$ and $\left(r_{0}=1 \mathrm{~cm}, \theta\right.$ changes $)$. Contrary to another experimental study for ZnSe:O by Kertzscher and Beddar (2019), we found that the absorbeddose energy response is not linear. It depends on not only the radial distance $r_{0}$ but also on the polar angle $\theta$. It is explained by the source anisotropy effect on the energy deposition in ZnSe which is more sensitive to changes in photon spectrum compared to water. That is, when the ratios of $\bar{D}_{\text {det }}$ and $D_{\text {wat }}$ are taken at a given position $\left(r_{0}, \theta\right)$, the source anisotropy effect does not cancel out. As a result, the absorbed-dose energy dependence correction factor can have multiple values at the same radial distance $r$. The findings demonstrate that a single absorbed-dose energy correction function is not sufficient for high- $Z$ detectors although it is adopted in some experimental studies (e.g., Ruiz-Arrebola et al 2020, Debnath et al 2021).

A good agreement between MC results and experimental data indicates that the assumption of $R_{Q_{0}, Q}$ being unity applies to ZnSe:O but it might be not the case for other detectors. In general, it would be of further interest to calibrate detectors in terms of $N_{D \text {, wat, } Q_{0}}$ with traceability to primary standards of high-energy external photon beams. It would require determination of the relative intrinsic energy dependence $R_{Q_{0}, Q}$ for measurements with ${ }^{192}$ Ir sources but would give a better understanding of detector response in low energy photon beams and would allow for independent determination of the absorbed dose to water as we presented in Paper I and Paper II. The results of Paper IV raise awareness to how in vivo detectors are characterized and which aspects should be considered. While the findings are most applicable to high- $Z$ detectors, not relying on TPS calculations and using MC simulations for the determination of absorbed-dose energy dependence would improve dosimetry practice and accuracy even for water-equivalent detectors, such as organic scintillators. We identified that a unified approach for dosimetry formalism is needed in the in vivo dosimetry field for a meaningful comparison of detector performance and for setting a reliable treatment error detection level. 



\section{CHAPTER}

\section{Conclusions and outlook}

The thesis addressed a two-fold problem in the experimental ${ }^{192}$ Ir BT dosimetry. Firstly, the absence of water-equivalent and well-characterized direct readout detectors that would enable regular in-phantom QA of BT treatment units. Secondly, incomplete characterization of already existing high atomic number inorganic scintillators used for in vivo dosimetry. Though the detectors and their applications are completely different, the underlying message of the thesis is to understand the detector at hand to enable development of high-accuracy experimental BT dosimetry.

Our proposed solution to the first problem is a synthetic diamond detector which was designed for small-field high-energy EBRT dosimetry. It has all necessary dosimetric properties though a more complicated structure and design than the detectors traditionally used in BT. However, by combining experimental and $\mathrm{MC}$ methods for detector characterization, we showed that it is suitable for non-relative dosimetry of $\mathrm{HDR}{ }^{192} \mathrm{Ir}$ sources with the uncertainties comparable to or lower than those of the currently used passive readout detectors. The detector had substantial intrinsic energy dependence in low energy photon beams relative to the high-energy calibration beam. It does not affect detector use for ${ }^{192}$ Ir BT dosimetry but may compromise nonrelative dosimetry of low-energy BT sources or electronic BT beams if not accounted for.

The continued investigation of synthetic diamond detectors resulted in contributions to not only BT but also to EBRT dosimetry where the detector was borrowed from. We clarified which diamond material properties (mass density, mean excitation energy, number of conduction electrons per atom) shall be used for MC simulations and calculations of mass electronic stopping powers. Previously, unawareness to differences in the dielectric response function of graphite and diamond has led to modelling of graphite and diamond 
hybrid in most studies on the diamond detector response. From the practical point of view, the change in energy deposition in diamond compared with the currently assumed pseudo-diamond was marginal, but the work highlights the importance of understanding the fundamental properties of detector medium and what lies behind correction factors calculated using MC simulations.

In-phantom BT dosimetry studies, including our work on synthetic diamond detectors, often combine experiments with MC simulations for detector characterization. While the limitations of MC methods must be considered, it is the most accurate method for the absorbed-dose energy dependence determination. However, its advantages are not exploited in in vivo dosimetry where experimental characterization using clinical sources and TPSs is prevalent. In the thesis we identified what errors may arise due to such approach and how the accuracy of detector characterization, and subsequently in vivo dosimetry, can be improved using MC methods. Detector calibration with traceability to primary standards of absorbed dose to water of high-energy EBRT could further improve in vivo dosimetry. This would require more extensive detector characterization but would enable independent check of treatment delivery which should be the primary aim.

While addressing practical issues related to the experimental BT dosimetry, the thesis also clarified how disperse are currently adopted practices. It would benefit the field to have a unified formalism and detector calibration practice. Currently, it is not straightforward to compare detectors and uncertainties associated with their performance either for in-phantom QA or in vivo dosimetry applications. 


\section{Bibliography}

Alfonso R, Andreo P, Capote R, Saiful Huq M, Kilby W, Kjäll P, Mackie T R, Palmans H, Rosser K, Seuntjens J, Ullrich W and Vatnitsky S 2008 A new formalism for reference dosimetry of small and nonstandard fields Med. Phys. 35 5179-86

Ali E S and Rogers D W O 2008 Benchmarking EGSnrc in the kilovoltage energy range against experimental measurements of charged particle backscatter coefficients Phys. Med. Biol. 53 1527-43

Almaviva S, Marinelli M, Milani E, Prestopino G, Tucciarone A, Verona C, Verona-Rinati G, Angelone M, Pillon M, Dolbnya I, Sawhney K and Tartoni N 2010 Chemical vapor deposition diamond based multilayered radiation detector: Physical analysis of detection properties J. Appl. Phys. 107014511

Almaviva S, Marinelli M, Milani E, Tucciarone A, Verona-Rinati G, Consorti R, Petrucci A, De Notaristefani F and Ciancaglioni I 2008 Synthetic single crystal diamond diodes for radiotherapy dosimetry Nucl. Instrum. Methods Phys. Res Nucl. Instrum. Methods Phys. Res. 594 273-7

Almond P R, Biggs P J, Coursey B M, Hanson W F, Saiful Huq M, Nath R and Rogers D W O 1991 AAPM's TG-51 protocol for clinical reference dosimetry of high-energy photon and electron beams Med. Phys. 26 1847-70

Andersen C E, Nielsen S K, Greilich S, Helt-Hansen J, Lindegaard J C and Tanderup K 2009a Characterization of a fiber-coupled $\mathrm{Al}_{2} \mathrm{O}_{3}$ :C luminescence dosimetry system for online in vivo dose verification during ${ }^{192}$ Ir brachytherapy Med. Phys. 36 708-18

Andreo P 2018 Monte Carlo simulations in radiotherapy dosimetry Radiat. Oncol. 13121

Andreo P and Benmakhlouf H 2017 Role of the density, density effect and mean excitation energy in solid-state detectors for small photon fields Phys. Med. Biol. 62 1518-32

Andreo P, Burns D T, Hohlfeld K, Saiful Huq M, Kanai T, Laitano F, Smyth V and Vynckier S 2000 Absorbed Dose Determination in External Beam Radiotherapy: An International Code of Practice for Dosimetry Based on Standards of Absorbed Dose to Water Technical Report Series No. 398 (Vienna: International Atomic Energy Agency)

Andreo P, Burns D T, Nahum A E, Seuntjens J and Attix F H 2017 Fundamentals of Ionizing Radiation Dosimetry (Hoboken, NJ: John Wiley \& Sons)

Andreo P, Burns D T and Salvat F 2012 On the uncertainties of photon mass energyabsorption coefficients and their ratios for radiation dosimetry Phys. Med. Biol. 57 $2117-36$

Andreo P, Palmans H, Marteinsdóttir M, Benmakhlouf H and Carlsson Tedgren Å 2016 On the Monte Carlo simulation of small-field micro-diamond detectors for megavoltage photon dosimetry Phys. Med. Biol. 61 L1-L10 
Anton M, Hackel T, Zink K, von Voigts-Rhetz P and Selbach H J 2015 Response of the alanine/ESR dosimeter to radiation from an Ir-192 HDR brachytherapy source Phys. Med. Biol. 60 175-93

Antonovic L, Gustafsson H, Alm Carlsson G and Carlsson Tedgren Å 2009 Evaluation of a lithium formate EPR dosimetry system for dose measurements around ${ }^{192} \mathrm{Ir}$ brachytherapy sources Med. Phys. 36 2236-47

Archambault L, Therriault-Proulx F, Beddar S and Beaulieu L 2012 A mathematical formalism for hyperspectral, multipoint plastic scintillation detectors Phys. Med. Biol. 57 7133-45

Beaulieu L and Beddar S 2016 Review of plastic and liquid scintillation dosimetry for photon, electron, and proton therapy Phys. Med. Biol. 61 R305-R343

Beaulieu L, Carlsson Tedgren A, Carrier J F, Davis S D, Mourtada F, Rivard M J, Thomson R M, Verhaegen F, Wareing T A and Williamson J F 2012 Report of the Task Group 186 on model-based dose calculation methods in brachytherapy beyond the TG-43 formalism: Current status and recommendations for clinical implementation Med. Phys. 39 6208-36

Benmakhlouf H, Johansson J, Paddick I and Andreo P 2015 Monte Carlo calculated and experimentally determined output correction factors for small field detectors in Leksell Gamma Knife Perfexion beams Phys. Med. Biol. 60 3959-73

Berger M J, Coursey B M, Zucker M A and Chang J 2017 ESTAR, PSTAR, and ASTAR: Computer Programs for Calculating Stopping-Power and Range Tables for Electrons, Protons, and Helium Ions (version 2.0.1) [Online] Available: http://physics.nist.gov/Star [accessed 15 September 2020] (Gaithersburg, MD: National Institute of Standards and Technology)

Büermann L, Grosswendt B, Kramer H M, Selbach H J, Gerlach M, Hoffmann M and Krumrey M 2006 Measurement of the x-ray mass energy-absorption coefficient of air using $3 \mathrm{keV}$ to $10 \mathrm{keV}$ synchrotron radiation Phys. Med. Biol. 51 5125-50

Canali C, Gatti E, Kozlov S F, Manfredi P F, Manfredotti C, Nava F and Quirini A 1978 Electrical properties and performances of natural diamond nuclear radiation detectors Nucl. Instrum. Methods $16073-7$

Carlsson Tedgren A, Elia R, Hedtjärn H, Olsson S and Alm Carlsson G 2012 Determination of absorbed dose to water around a clinical HDR ${ }^{192} \mathrm{Ir}$ source using LiF:Mg, Ti TLDs demonstrates an LET dependence of detector response Med. Phys. 39 1133-40

Carlsson Tedgren Å, Hedman A, Grindborg J E and Alm Carlsson G 2011 Response of $\mathrm{LiF}: \mathrm{Mg}, \mathrm{Ti}$ thermoluminescent dosimeters at photon energies relevant to the dosimetry of brachytherapy (<1 MeV) Med. Phys. 38 5539-50

Ciancaglioni I, Marinelli M, Milani E, Prestopino G, Verona C, Verona-Rinati G, Consorti R, Petrucci A and De Notaristefani F 2012 Dosimetric characterization of a synthetic single crystal diamond detector in clinical radiation therapy small photon beams $M e d$. Phys. 39 4493-501

Damodar J, Odgers D, Pope D and Hill R 2018 A study on the suitability of the PTW microDiamond detector for kilovoltage x-ray beam dosimetry Appl. Radiat. Isot. 135 104-9

Davis S D, Ross C K, Mobit P N, Van der Zwan L, Chase W J and Shortt K R 2003 The response of $\mathrm{LiF}$ thermoluminescence dosemeters to photon beams in the energy range from $30 \mathrm{kV}$ X rays to ${ }^{60} \mathrm{Co}$ gamma rays Radiat. Prot. Dosimetry $10633-43$

De Angelis C, Casati M, Bruzzi M, Onori S and Bucciolini M 2007 Present limitations of CVD diamond detectors for IMRT applications Nucl. Instr. Methods Phys. Res. 583 195-203 
De Angelis C, Onori S, Pacilio M, Cirrone G A, Cuttone G, Raffaele L, Bucciolini M and Mazzocchi S 2002 An investigation of the operating characteristics of two PTW diamond detectors in photon and electron beams Med. Phys. 29 248-54

deAlmeida C E, Ochoa R, Lima M C, David M G, Pires E J, Peixoto J G, Salata C and Bernal M A 2014 A feasibility study of Fricke dosimetry as an absorbed dose to water standard for ${ }^{192}$ Ir HDR sources Plos One 9 e115155

Debnath S B C, Ferre M, Tonneau D, Fauquet C, Tallet A, Goncalves A and Darreon J 2021 High resolution small-scale inorganic scintillator detector: HDR brachytherapy application Med. Phys. doi:10.1002/MP.14727

DeWerd L A, Ibbott G S, Meigooni A S, Mitch M G, Rivard M J, Stump K E, Thomadsen B R and Venselaar J L 2011 A dosimetric uncertainty analysis for photon-emitting brachytherapy sources: Report of AAPM Task Group No. 138 and GEC-ESTRO Med. Phys. 38 782-801

Eaton D J 2015 Electronic brachytherapy-current status and future directions $\mathrm{Br}$. J. Radiol. 8820150002

Famulari G, Duclos M and Enger S A 2020 A novel ${ }^{169}$ Yb-based dynamic-shield intensity modulated brachytherapy delivery system for prostate cancer Med. Phys. 47 859-68

Fano U 1963 Penetration of protons, alpha particles and mesons Ann. Rev. Nucl. Sci. 13 $1-66$

Fernández-Varea J M, Górka B and Nilsson B 2021 Electronic stopping power of electrons in diamond Phys. Med. Biol. (submitted)

Fonseca G P, Johansen J G, Smith R L, Beaulieu L, Beddar S, Kertzscher G, Verhaegen F and Tanderup K 2020 In vivo dosimetry in brachytherapy: Requirements and future directions for research, development, and clinical practice Phys. Imag. Radiat. Oncol. 16 1-11

Fonseca G P, Podesta M, Bellezzo M, Van den Bosch M R, Lutgens L, Vanneste B G L, Voncken R, Van Limbergen E J, Reniers B and Verhaegen F 2017 Online pretreatment verification of high-dose rate brachytherapy using an imaging panel Phys. Med. Biol. 62 5440-61

Fowler J F 1966 Solid state electrical conductivity dosimeters (Radiation Dosimetry vol 2) ed F H Attix F H and W C Roesch (New York: Academic Press)

Frenière N 2018 COMP report: CPQR technical quality control guidelines for brachytherapy remote afterloaders J. Appl. Clin. Med. Phys. 19 39-43

Goetsch S J, Attix F H, Pearson D W and Thomadsen B R 1991 Calibration of ${ }^{192}$ Ir high-dose-rate afterloading systems Med. Phys. 18 462-7

Górka B, Nilsson B, Svensson R, Brahme A, Ascarelli P, Trucchi D M, Conte G and Kalish R 2008 Design and characterization of a tissue-equivalent CVD-diamond detector for clinical dosimetry in high-energy photon beams Phys. Med. 24 159-68

Granero D, Vijande J, Ballester F and Rivard M J 2011 Dosimetry revisited for the HDR ${ }^{192}$ Ir brachytherapy source model mHDR-v2 Med. Phys. 38 487-94

Hartmann G H, Zink K 2019 A Monte Carlo study on the PTW 60019 microDiamond detector Med. Phys. 46 5159-72

ICRU 1984 Stopping Powers for Electrons and Positrons ICRU Report 37 (Bethesda, MD: International Commission on Radiation Units and Measurements)

ICRU 1985 Dose and Volume Specification for Reporting Intracavitary Therapy in Gynaecology ICRU Report 38 (Bethesda, MD: International Commission on Radiation Units and Measurements) 
ICRU 1997 Dose and Volume Specification for Reporting Interstitial Therapy ICRU Report 58 (Bethesda, MD: International Commission on Radiation Units and Measurements)

ICRU 2016 Key Data for Ionizing-Radiation Dosimetry: Measurement Standards and Applications ICRU Report 90 (Bethesda, MD: International Commission on Radiation Units and Measurements)

Johansen J G, Rylander S, Buus S, Bentzen L, Hokland S B, Sondergaard C S, With A K M, Kertzscher G and Tanderup K 2018 Time-resolved in vivo dosimetry for source tracking in brachytherapy Brachytherapy 17 122-32

Jørgensen E B, Johansen J G, Overgaard J, Piché-Meunier D, Tho D, Linares Rosales H M, Beaulieu L, Kertzscher G and Beddar A S 2021a A novel inorganic scintillator-based detector system for time-resolved in vivo dosimetry during brachytherapy Manuscript in preparation

Jørgensen E B, Kertzscher G, Buus S, Bentzen L, Hokland S B, Rylander S, Tanderup $\mathrm{K}$ and Johansen J G 2021b Accuracy of an in vivo dosimetry based source tracking method for afterloaded brachytherapy - A phantom study Med. Phys. 55 doi.org/10.1002/mp.14812

Kawrakow I, Mainegra-Hing E, Rogers D W O, Tessier F and Walters B R B 2013 The EGSnrc Code System: Monte Carlo Simulation of Electron and Photon Transport NRCC Report PIRS 701 (Ottawa: National Research Council)

Kertzscher G, Andersen C E and Tanderup K 2014a Fast, Simple, and Accurate: In Vivo Dosimeter Calibration for HDR/PDR Brachytherapy Int. J. Radiat. Oncol. $90 \mathrm{~S} 742$

Kertzscher G and Beddar S 2017 Inorganic scintillation detectors based on Eu-activated phosphors for ${ }^{192}$ Ir brachytherapy Phys. Med. Biol. 62 5046-75

Kertzscher G and Beddar S 2019 Inorganic scintillation detectors for ${ }^{192}$ Ir brachytherapy Phys. Med. Biol. 64225018

Kertzscher G, Rosenfeld A, Beddar S, Tanderup K and Cygler J E 2014b In vivo dosimetry: trends and prospects for brachytherapy Br. J. Radiol. 8720140206

Khan A U, Culberson W S and DeWerd L A 2020 Characterizing a PTW microDiamond detector in kilovoltage radiation beams Med. Phys. 47 4553-62

Kozlov S F, Stuck R, Hage-Ali M and Siffert P 1975 Preparation and characteristics of natural diamond nuclear radiation detectors IEEE. Trans. Nucl. Sci. 22 160-70

Kubo H, Glasgow G P, Pethel T D, Thomadsen B R and Williamson J F 1998 High doserate brachytherapy treatment delivery: Report of the AAPM Radiation Therapy Committee Task Group No. 59 Med. Phys. 25 375-403

Lambert J, McKenzie D R, Law S, Elsey J and Suchowerska N 2006 A plastic scintillation dosimeter for high dose rate brachytherapy Phys. Med. Biol. 51 5505-16

Lárraga-Gutiérrez J M, Ballesteros-Zebadúa P, Rodríguez-Ponce M, García-Garduño O A and G'alvan de la Cruz O O 2015 Properties of a commercial PTW-60019 synthetic diamond detector for the dosimetry of small radiotherapy beams Phys. Med. Biol. 60 905-24

Laub W U 2002 Comparison of TG-43 dose calculations to pinpoint ion chamber and diamond detector measurements Phys. Med. Biol. 47 315-8

Laub W U and Crilly R 2014 Clinical radiation therapy measurements with a new commercial synthetic single crystal diamond detector J. Appl. Clin. Med. Phys. 15 $1-11$

Laub W U, Kaulich T W and Nüsslin F 1997 Energy and dose rate dependence of a diamond detector in the dosimetry of 4-25 MV photon beams Med. Phys. 24 535-6 
Li Z, Das R K, DeWerd L A, Ibbott G S, Meigooni A S, Pérez-Calatayud J, Rivard M J, Sloboda R S and Williamson J F 2007 Dosimetric prerequisites for routine clinical use of photon emitting brachytherapy sources with average energy higher than 50 keV Med. Phys. 34 37-40

Linares Rosales H M, Archambault L, Beddar S and Beaulieu L 2020a Dosimetric performance of a multipoint plastic scintillator dosimeter as a tool for real-time source tracking in high dose rate ${ }^{192}$ Ir brachytherapy Med. Phys. 47 4477-90

Linares Rosales H M, Duguay-Drouin P, Archambault L, Beddar S and Beaulieu L 2019 Optimization of a multipoint plastic scintillator dosimeter for high dose rate brachytherapy Med. Phys. 46 2412-21

Linares Rosales H M, Johansen J G, Kertzscher G, Tanderup K, Beaulieu L and Beddar S 2020b 3D source tracking and error detection in HDR using two independent scintillator dosimetry systems Med. Phys. doi.org/10.1002/mp.14607

Mainegra-Hing E and Rogers D W O 2006 On the accuracy of techniques for obtaining the calibration coefficient $N_{K}$ of ${ }^{192}$ Ir HDR brachytherapy sources Med. Phys. 33 $3340-7$

Marinelli M, Prestopino G, Verona C and Verona-Rinati G 2016 Experimental determination of the PTW 60019 microDiamond dosimeter active area and volume Med. Phys. 43 5205-12

Marsolat F, Tromson D, Tranchant N, Pomorski M, Bassinet C, Huet C, Derreumaux S, Chea M, Cristina K, Boisserie G, Buchheit I, Marchesi V, Gaudaire-Josset S, Lisbona A, Lazaro D, Hugon R and Bergonzo P 2015 Why diamond dimensions and electrode geometry are crucial for small photon beam dosimetry J. Appl. Phys. 118234507

Mobit P N, Nahum A E and Mayles P 1997 A Monte Carlo study of the quality dependence of diamond thermoluminescent dosimeters in radiotherapy beams Phys. Med. Biol. 42 1913-27

Nunn A A, Davis S D, Micka J A and DeWerd L A 2008 LiF:Mg,Ti TLD response as a function of photon energy for moderately filtered x-ray spectra in the range of 20-250 $\mathrm{kVp}$ relative to ${ }^{60} \mathrm{Co}$ Med. Phys. 35 1859-69

Olko P, Bilski P and Kim J L 2002 Microdosimetric interpretation of the photon energy response of LiF:Mg,Ti Radiat. Prot. Dosimetry 100 119-22

Pérez-Calatayud J, Ballester F, Das R K, Dewerd L A, Ibbott G S, Meigooni A S, Ouhib Z, Rivard M J, Sloboda R S and Williamson J F 2012 Dose calculation for photonemitting brachytherapy sources with average energy higher than $50 \mathrm{keV}$ : Report of the AAPM and ESTRO Med. Phys. 39 2904-29

Poder J, Cutajar D, Guatelli S, Petasecca M, Howie A, Bucci J, Carrara M and Rosenfeld A 2019 A Monte Carlo study on the feasibility of real-time in vivo source tracking during ultrasound based HDR prostate brachytherapy treatments Phys. Med. 59 $30-6$

Qi Z Y, Deng X W, Huang S M, Lu J, Lerch M, Cutajar D and Rosenfeld A 2007 Verification of the plan dosimetry for high dose rate brachytherapy using metal-oxidesemiconductor field effect transistor detectors Med. Phys. 34 2007-13

Quast U, Kaulich T W, Álvarez-Romero J T, Carlsson Tedgren Å, Enger S A, Medich D C, Mourtada F, Pérez-Calatayud J, Rivard M J and Zakaria G A 2016 A brachytherapy photon radiation quality index $\mathrm{Q}^{\mathrm{BT}}$ for probe-type dosimetry Phys. Med. 32 741-8

Raffi J A, Davis S D, Hammer C G, Micka J A, Kunugi K A, Musgrove J E, Winston J W, Jr., Ricci-Ott T J and DeWerd L A 2010 Determination of exit skin dose for ${ }^{192}$ Ir intracavitary accelerated partial breast irradiation with thermoluminescent dosimeters Med. Phys. 7 2693-702 
Rassmussen B E, Davis S D, Schmidt C R and Micka J A 2011 Comparison of air-kerma strength determinations for HDR ${ }^{192}$ sources Med. Phys. 38 6721-9

Rivard M J, Coursey B M, DeWerd L A, Hanson W F, Huq M S, Ibbott G S, Mitch M G, Nath R and Williamson J F 2004 Update of AAPM Task Group No. 43 Report: A revised AAPM protocol for brachytherapy dose calculations Med. Phys. 31 633-74

Rossi G, Gainey M, Kollefrath M, Hofmann E and Baltas D 2020 Suitability of the microDiamond detector for experimental determination of the anisotropy function of High Dose Rate ${ }^{192}$ Ir brachytherapy sources Med. Phys. 47 5838-51

Rossi G, Gainey M, Thomann B, Kollefrath M, Würfel J, Allgaier B and Baltas D 2019 Monte Carlo and experimental high dose rate ${ }^{192}$ Ir brachytherapy dosimetry with microDiamond detectors Z. Med. Phys. 29 272-81

Ruiz-Arrebola S, Fabregat-Borrás R, Rodríguez E, Fernández-Montes M, Pérez-Macho M, Ferri M, García A, Cardenal J, Pacheco M T, Anchuelo J, Tornero-Lopez A M, Prada P J and Guirado D 2020 Characterization of microMOSFET detectors for in vivo dosimetry in high-dose-rate brachytherapy with ${ }^{192} \mathrm{Ir}$ Med. Phys. 47 2242-53

Rustgi S N 1998 Application of a diamond detector to brachytherapy dosimetry Phys. Med. Biol. 43 2085-94

Salvat F 2019 PENELOPE-2018: A Code System for Monte Carlo Simulation of Electron and Photon Transport (Issy-les-Moulineaux: OECD Nuclear Energy Agency)

Sander T and Nutbrown R F 2006 The NPL air kerma primary standard TH100C for high dose rate ${ }^{192}$ Ir brachytherapy sources NPL Report DQL-RD 004 (Teddington: National Physics Laboratory)

Santiago Ayala Alvarez D, Watson P G F, Popovic M, Jean Heng V, Evans M D C and Seuntjens J 2020 Monte Carlo calculation of the relative TG-43 dosimetry parameters for the INTRABEAM electronic brachytherapy source Phys. Med. Biol. 65245041

Sarfehnia A and Seuntjens J 2010 Development of a water calorimetry-based standard for absorbed dose to water in HDR ${ }^{192}$ Ir brachytherapy Med. Phys. 37 1914-23

Sempau J, Badal A and Brualla L 2011 A PENELOPE-based system for the automated Monte Carlo simulation of clinacs and voxelized geometries-application to far-fromaxis fields Med. Phys. 38 5887-95

Sempau J, Fernández-Varea J M, Acosta E and Salvat F 2003 Experimental benchmarks of the Monte Carlo code PENELOPE Nucl. Instr. Methods Phys. Res. 207 107-23

Smith R L, Hanlon M, Panettieri V, Millar J L, Matheson B, Haworth A and Franich R D 2018 An integrated system for clinical treatment verification of HDR prostate brachytherapy combining source tracking with pretreatment imaging Brachytherapy 17 111-21

Soares C G, Douysset G and Mitch M G 2009 Primary standards and dosimetry protocols for brachytherapy sources Metrologia 46 S80-S98

Steenhujsen J, Harbers M, Hoffmann A, de Leeuw A, Rijnders A and Unipan M 2018 Code of Practice for Quality Assurance of Brachytherapy with Ir-192 Afterloaders Report 30 (Delft: The Netherlands Commission on Radiation Dosimetry)

Tanderup K, Beddar S, Andersen C E, Kertzscher G and Cygler J E 2013 In vivo dosimetry in brachytherapy Med. Phys. 40070902

Taylor R E P and Rogers D W O 2008 EGSnrc Monte Carlo calculated dosimetry parameters for ${ }^{192} \mathrm{Ir}$ and ${ }^{169} \mathrm{Yb}$ brachytherapy sources Med. Phys. 35 4933-44

Therriault-Proulx F, Briere T M, Mourtada F, Aubin S, Beddar S and Beaulieu L 2011 A phantom study of an in vivo dosimetry system using plastic scintillation detectors for real-time verification of ${ }^{192}$ Ir HDR brachytherapy Med. Phys. 38 2542-51 
Thomson R M and Kawrakow I 2011 On the Monte Carlo simulation of electron transport in the sub-1 keV energy range Med. Phys. 38 4531-4

Valdes-Cortez C, Mansour I, Rivard M J, Ballester F, Mainegra-Hing E, Thomson R M and Vijande J 2021 A study of Type B uncertainties associated with the photoelectric effect in low-energy Monte Carlo simulations Phys. Med. Biol. doi:10.1088/1361$6560 /$ abebfd

Venselaar J and Pérez-Calatayud J 2004 A Practical Guide to Quality Control of Brachytherapy Equipment ESTRO Booklet No. 8 (Brussels: European Society for Therapeutic Radiology and Oncology)

Westermark M, Arndt J, Nilsson B and Brahme A 2000 A comparative dosimetry in narrow high-energy photon beams Phys. Med. Biol. 45 685-702

Zilio V O, Joneja O P, Popowski Y, Rosenfeld A and Chawla R 2006 Absolute depth-doserate measurements for an ${ }^{192}$ Ir HDR brachytherapy source in water using MOSFET detectors Med. Phys. 33 1532-9 



\section{Papers}

The papers associated with this thesis have been removed for copyright reasons. For more details about these see:

http://urn.kb.se/resolve?urn=urn:nbn:se:liu:diva-175192 


\section{FACULTY OF MEDICINE AND HEALTH SCIENCES}

Linköping University Medical Dissertation No. 1775, 2021

Department of Health, Medicine and Caring Sciences

Linköping University

SE-581 83 Linköping, Sweden

www.liu.se 